\title{
Thermal desorption of circumstellar and cometary ice analogs
}

\author{
R. Martín-Doménech ${ }^{1}$, G.M. Muñoz Caro ${ }^{1}$, J. Bueno ${ }^{2}$, and F. Goesmann ${ }^{3}$ \\ ${ }^{1}$ Centro de Astrobiología (INTA-CSIC), Ctra. de Ajalvir, km 4, Torrejón de Ardoz, 28850 Madrid, Spain \\ e-mail: rmartin@cab.inta-csic.es \\ 2 Netherlands Institute for Space Research, Sorbonnelaan 2, 3584 CA Utrecht, Netherlands \\ 3 Max Planck Institute for Solar System Research, Justus von Liebig Weg 3, 370077 Göttingen, Germany
}

Received 10 October 2013 / Accepted 11 February 2014

\begin{abstract}
Context. Thermal annealing of interstellar ices takes place in several stages of star formation. Knowledge of this process comes from a combination of astronomical observations and laboratory simulations under astrophysically relevant conditions.

Aims. For the first time we present the results of temperature programmed desorption (TPD) experiments with pre-cometary ice analogs composed of up to five molecular components: $\mathrm{H}_{2} \mathrm{O}, \mathrm{CO}, \mathrm{CO}_{2}, \mathrm{CH}_{3} \mathrm{OH}$, and $\mathrm{NH}_{3}$.

Methods. The experiments were performed with an ultra-high vacuum chamber. A gas line with a novel design allows the controlled preparation of mixtures with up to five molecular components. Volatiles desorbing to the gas phase were monitored using a quadrupole mass spectrometer, while changes in the ice structure and composition were studied by means of infrared spectroscopy.

Results. The TPD curves of water ice containing $\mathrm{CO}, \mathrm{CO}_{2}, \mathrm{CH}_{3} \mathrm{OH}$, and $\mathrm{NH}_{3}$ present desorption peaks at temperatures near those observed in pure ice experiments, volcano desorption peaks after water ice crystallization, and co-desorption peaks with water. Desorption peaks of $\mathrm{CH}_{3} \mathrm{OH}$ and $\mathrm{NH}_{3}$ at temperatures similar to the pure ices takes place when their abundance relative to water is above $\sim 3 \%$ in the ice matrix. We found that $\mathrm{CO}, \mathrm{CO}_{2}$, and $\mathrm{NH}_{3}$ also present co-desorption peaks with $\mathrm{CH}_{3} \mathrm{OH}$, which cannot be reproduced in experiments with binary water-rich ice mixtures. These are extensively used in the study of thermal desorption of interstellar ices.

Conclusions. These results reproduce the heating of circumstellar ices in hot cores and can be also applied to the late thermal evolution of comets. In particular, TPD curves represent a benchmark for the analysis of the measurements that mass spectrometers on board the ESA-Rosetta cometary mission will perform on the coma of comet 67P/Churyumov-Gerasimenko, which will be active before the arrival of Rosetta according to our predictions
\end{abstract}

Key words. ISM: molecules - circumstellar matter - comets: general - methods: laboratory: solid state - techniques: spectroscopic

\section{Introduction}

Dense molecular clouds have typical densities of $10^{4}-10^{6}$ particles $\mathrm{cm}^{-3}$ and temperatures down to $10 \mathrm{~K}$ in their interiors. These low temperatures are reached thanks to the screening from the interstellar ultraviolet (UV) radiation field provided by the gas, dust particles and, presumably, polycyclic aromatic hydrocarbons (PAHs) at the edge of the cloud that absorb most of the radiation. Under these conditions, molecules are able to condense onto the surface of dust grains, forming ice mantles. The size of interstellar dust grains is usually given by dust models as a power-law distribution, covered by a $\sim 0.01 \mu \mathrm{m}$ ice mantle in dense cloud interiors (Zubko et al. 2004).

When a dense core collapses to form a star, grain agglomeration can take place during the cold core phase, leading to cluster particles composed of sub-micron grains. Later on, during the warm-up phase, the protostar heats its environment, leading to thermal processing of ice mantles. This thermal annealing takes place in two different ways. On the one hand, grains at a certain distance are heated (approximately $1 \mathrm{~K} /$ century; Viti \& Williams 1999) as the temperature of the protostar increases. On the other hand, grains are also able to undergo periodic radial excursions to distances less than $0.1 \mathrm{AU}$ from the central protostar on short timescales (from a few to hundreds of hours), according to the fluctuating X-wind model for the formation of $\mathrm{Ca}$-Al-rich inclusions (CAIs) and chondrules (Shu et al. 1996, 1997, 2001).
The main effect of thermal annealing is the sublimation of ice mantles explaining the excess gas-phase abundances of some species in the inner regions of protostellar envelopes (e.g., Pauls et al. 1983; Herbst \& van Dischoeck 2009, and references therein). Models predict that thermal desorption of ice governs the gas-phase chemistry during star formation (e.g., Viti et al. 2004).

The remaining ice mantles in protoplanetary disks are incorporated in comets and other minor bodies. Cometary ice composition is thought to be similar to that of the interstellar ice mantles (Mumma \& Charnley 2011, and references therein), although it can vary between comets. Molecules present on ices, both originally or created afterwards by means of energetic processing, are thus transported by these bodies, which may be responsible for the delivery of organic material to habitable planets, and, ultimately, the origin of life.

Cometary ices are also exposed to thermal processing, which is the main responsible for the activity observed in comets. When a comet passes within $r \sim 3 \mathrm{AU}$ of the Sun during its orbit, icy volatiles form an expanding atmosphere of gas and dust called the coma (Wyckof 1982).

Most of our current knowledge on interstellar, circumstellar, and cometary ices comes from a combination of infrared (IR) observations and laboratory experiments that simulate their energetic processing under astrophysically relevant conditions. Heating of ice mixtures leads to structural changes in the 
ice, involving diffusion of molecules, phase changes, and ice segregation. Segregation is expected to occur whenever ice diffusion of molecules is possible, and it is energetically favorable for molecules of the same kind to group together. Ice diffusion barriers are proportional to the binding energy of the species (i.e., their volatility). Thermal annealing of ice samples also leads to sequential desorption of ice molecules that are able to diffuse through the structure of the ice toward the surface, which starts with the most volatile species (Öberg et al. 2009).

The experiments presented here contribute to a better understanding on the thermal processing of circumstellar and cometary ices. The majority of published results of temperature programmed desorption (TPD) experiments of astrophysical ice analogs deal with pure ices or binary mixtures with $\mathrm{H}_{2} \mathrm{O}$ as the primary component (e.g., Collings et al. 2004). Some ternary mixtures have been treated as well (e.g., Fayolle et al. 2011).

This work represents the first attempt to study the thermal processing of a more realistic astrophysical ice with up to five molecular components $\left(\mathrm{H}_{2} \mathrm{O}, \mathrm{CO}, \mathrm{CO}_{2}, \mathrm{CH}_{3} \mathrm{OH}\right.$, and $\left.\mathrm{NH}_{3}\right)$ using both mass spectrometry and IR spectroscopy.

Our results can also be used to predict and analyze the data collected by mass spectrometers on board cometary missions like Rosetta. The International Rosetta Mission was approved in 1993 and has been developed by the European Space Agency (ESA). Since its approval, several works have been published studying the target of this cometary mission using both ground and space-based observations with special interest on the evolution of the activity during its orbit (e.g., Kelley et al. 2009; de Almeida et al. 2009; Snodgrass et al. 2013). Rosetta was launched in March 2004 and will arrive at comet 67P/Churyumov-Gerasimenko (a short-period comet with $P=$ $6.45 \mathrm{yrs}$ ) in July 2014 at $\sim 3.8$ AU from the Sun. The Rosetta orbiter will remain in close proximity to the comet nucleus, and a small lander will be released onto its surface. When the mission draws to a close in December 2015, the comet will be on its way out of the inner Solar System. Previous cometary missions have provided in situ measurements of cometary gas composition (e.g., for comet Halley; Eberhardt 1999). Ground-based observations have been also used to detect coma components (Mumma \& Charnley 2011, and references therein). However, no direct measurements of cometary ice composition are avaliable so far. The NASA-Deep Impact cometary mission studied the nucleus of comet 9P/Temple 1 but from a more geological point of view (e.g., Thomas et al. 2007). Rosetta instruments will provide for the first time simultaneous measurements of both the nucleus and the coma of a comet. Our experiments, using realistic astrophysical ice analogs, can be used to understand the relation between the nucleus and the coma compositions and also the cometary ice conditions. However, as we discuss, caution is needed to extrapolate these experimental results to a real cometary scenario.

The layout of this paper is as follows. In Sect. 2, we describe the experimental protocol. Section 3 presents the experimental results. Their astrophysical implications are discussed in Sect. 4. Section 5 summarizes the main conclusions.

\section{Experimental}

The results presented here have been obtained using the novel InterStellar Astrochemistry Chamber (ISAC) at the Centro de Astrobiología (Muñoz Caro et al. 2010). The ISAC set-up is an ultra-high vacuum (UHV) chamber with a pressure about $4 \times 10^{-11}$ mbar, which corresponds to a density of $10^{6} \mathrm{~cm}^{-3}$ (Muñoz Caro et al. 2010), similar to that found in dense cloud
Table 1. Infrared feature used to calculate the column density of each component (frequencies and band strengths for pure ices at $8 \mathrm{~K}$ ).

\begin{tabular}{cccc}
\hline \hline Molecule & $\begin{array}{c}\text { Vibrational } \\
\text { mode }\end{array}$ & $\begin{array}{c}\text { Frequency } \\
\left(\mathrm{cm}^{-1}\right)\end{array}$ & $\begin{array}{c}\text { Band strength } \\
\left.(\mathrm{cm} \mathrm{molec})^{-1}\right)\end{array}$ \\
\hline $\mathrm{H}_{2} \mathrm{O}$ & stretching O-H & 3280 & $2.0 \times 10^{-16 a}$ \\
$\mathrm{CO}$ & stretching $\mathrm{C}=\mathrm{O}$ & 2138 & $1.1 \times 10^{-17 b}$ \\
$\mathrm{CO}_{2}$ & stretching $\mathrm{C}=\mathrm{O}$ & 2344 & $7.6 \times 10^{-17 c}$ \\
$\mathrm{CH}_{3} \mathrm{OH}$ & stretching C-O & 1025 & $1.8 \times 10^{-17 d}$ \\
$\mathrm{NH}_{3}$ & umbrella mode & 1070 & $1.7 \times 10^{-17 e}$ \\
\hline
\end{tabular}

Notes. ${ }^{(a)}$ From Hagen (1981). ${ }^{(b)}$ From Jiang et al. (1975). ${ }^{(c)}$ From Yamada \& Person (1964). ${ }^{(d)}$ From d'Hendecourt \& Allamandola (1986). ${ }^{(e)}$ From Sandford \& Allamandola (1993).

interiors. Ice samples made by deposition of a gas mixture onto a $\mathrm{KBr}$ window at $8 \mathrm{~K}$ (achieved by means of a closed-cycle helium cyostat) were warmed up until a complete sublimation was attained. A silicon diode temperature sensor and a LakeShore Model 331 temperature controller are used, reaching a sensitivity of about $0.1 \mathrm{~K}$. Complex gas mixtures were prepared in the gas line system, using a Pfeiffer Prisma quadrupole mass spectrometer (QMS) of mass spectral range from 1 to $100 \mathrm{amu}$ with a Faraday detector, and electrovalves were used to control the flow of the components, thus allowing co-deposition of gas mixtures with the desired composition. A second deposition tube was used to introduce corrosive gases, such as $\mathrm{NH}_{3}$.

The chemical components used in the experiments were $\mathrm{H}_{2} \mathrm{O}$ (liquid), triply distilled, $\mathrm{CH}_{3} \mathrm{OH}$ (liquid, Panreac 99.9\%), $\mathrm{CO}$ (gas, Praxair 99.998\%), $\mathrm{CO}_{2}$ (gas, Praxair 99.996\%), and $\mathrm{NH}_{3}$ (gas, Praxair 99.999\%), which was deposited through the second deposition tube.

The evolution of the solid sample was monitored by in situ Fourier transform infrared (FTIR) transmittance spectroscopy, using a Bruker Vertex 70 spectrometer equipped with a deuterated triglycine sulfate detector (DTGS). The IR spectra were collected after ice deposition at $8 \mathrm{~K}$, or every five minutes during warm-up, with a spectral resolution of $2 \mathrm{~cm}^{-1}$. Column densities of each species in the ice were calculated from the IR spectra using the formula

$N=\frac{1}{A} \int_{\text {band }} \tau_{v} \mathrm{~d} v$

where $N$ is the column density in molecules $\mathrm{cm}^{-2}, \tau_{v}$ the optical depth of the absorption band, and $A$ the band strength in $\mathrm{cm}$ molecule $\mathrm{e}^{-1}$, as derived from laboratory experiments (Table 1). Band strengths were measured for pure ices made of one molecular component. The same values are usually adopted in ice mixtures, which introduce an uncertainty of about 20-30\% (d'Hendecourt \& Allamandola 1986).

The desorbing molecules were detected by a second Pfeiffer Prisma QMS of mass spectral range from 1 to $200 \mathrm{amu}$ with a Channeltron detector, which is situated $\sim 17 \mathrm{~cm}$ apart from the sample. Gas-phase molecules were ionized by low energy $(\sim 70 \mathrm{eV})$ electron bombardment. Every species was monitored through its main mass fragment (except for $\mathrm{NH}_{3}$ ), namely: $m / z=18\left(\mathrm{H}_{2} \mathrm{O}\right), m / z=28(\mathrm{CO}$, with a small contribution of $\mathrm{CO}_{2}$ fragmentation into $\mathrm{CO}^{+}$of, approximately, $10 \%$ of the $m / z=44$ signal $), m / z=31\left(\mathrm{CH}_{3} \mathrm{OH}\right.$, because the ion molecule with $\mathrm{m} / z=32$ coincides with $\mathrm{O}_{2}$ ), and $\mathrm{m} / z=44$ $\left(\mathrm{CO}_{2}\right)$. Ammonia, $\mathrm{NH}_{3}$, was monitored through the mass fragment $\mathrm{m} / \mathrm{z}=15$ to avoid confusion with the mass fragment $m / z=17$ of $\mathrm{H}_{2} \mathrm{O}$. The $m / z=17$ signal was, nonetheless, used 
Table 2. Composition of the ice mixture measured with FTIR spectroscopy at $8 \mathrm{~K}$.

\begin{tabular}{ccccccc}
\hline \hline Exp & $\begin{array}{c}\mathrm{H}_{2} \mathrm{O} \\
(\%)\end{array}$ & $\begin{array}{c}\mathrm{CO} \\
(\%)\end{array}$ & $\begin{array}{c}\mathrm{CO}_{2} \\
(\%)\end{array}$ & $\begin{array}{c}\mathrm{CH}_{3} \mathrm{OH} \\
(\%)\end{array}$ & $\begin{array}{c}\mathrm{NH}_{3} \\
(\%)\end{array}$ & $\begin{array}{c}N / 10^{15} \\
\mathrm{~mol} / \mathrm{cm}^{2}\end{array}$ \\
\hline $\mathrm{E}^{a}$ & 100.0 & 0.0 & 0.0 & 0.0 & 0.0 & 1554.61 \\
& 100.0 & 0.0 & 0.0 & 0.0 & 0.0 & \\
$\mathrm{E}^{a}$ & 0 & 100.0 & 0.0 & 0.0 & 0.0 & 104.97 \\
& 0.0 & 100.0 & 0.0 & 0.0 & 0.0 & \\
$\mathrm{E}^{a}$ & 0.0 & 0.0 & 100.0 & 0.0 & 0.0 & 134.88 \\
& 0.0 & 0.0 & 100.0 & 0.0 & 0.0 & \\
$\mathrm{E}^{a}$ & 0.0 & 0.0 & 0.0 & 100.0 & 0.0 & 190.81 \\
& 0.0 & 0.0 & 0.0 & 100.0 & 0.0 & \\
$\mathrm{E}^{a}$ & 0.0 & 0.0 & 0.0 & 0.0 & 100.0 & 154.60 \\
& 0.0 & 0.0 & 0.0 & 0.0 & 100.0 & \\
\hline $\mathrm{E}^{b}$ & 100.0 & 7.8 & 6.8 & 1.6 & 0.0 & 308.69 \\
& 86.1 & 6.7 & 5.8 & 1.3 & 0.0 & \\
$\mathrm{E}^{b}$ & 100.0 & 6.6 & 27.9 & 3.1 & 3.4 & 1395.54 \\
& 70.9 & 4.7 & 19.8 & 2.2 & 2.4 & \\
$\mathrm{E}^{b}$ & 100.0 & 0.0 & 0.0 & 9.3 & 0.0 & 109.52 \\
& 91.5 & 0.0 & 0.0 & 8.5 & 0.0 & \\
$\mathrm{E}^{b}$ & 100.0 & 0.0 & 0.0 & 68.6 & 0.0 & 385.58 \\
& 59.3 & 0.0 & 0.0 & 40.7 & 0.0 & \\
\hline $\mathrm{E} 10^{b}$ & 100.0 & 8.7 & 20.2 & 5.7 & 5.7 & 749.50 \\
& 71.2 & 6.2 & 14.4 & 4.1 & 4.1 & \\
$\mathrm{E} 11^{a}$ & 100.0 & 15.0 & 10.3 & 7.2 & 8.0 & 912.43 \\
& 71.2 & 10.7 & 7.3 & 5.7 & 5.1 & \\
\hline
\end{tabular}

Notes. For each experiment, the first row indicates the abundance (by number of molecules) in percent relative to water, while the second row shows the absolute abundance (also in percent). ${ }^{(a)}$ Heating rate $=$ $1 \mathrm{~K} / \mathrm{min} .{ }^{(b)}$ Heating rate $=2 \mathrm{~K} / \mathrm{min}$.

to confirm that the $m / z=15$ signal was not produced by contaminants present in small amounts.

An overview of all the experiments is shown in Table 2. All ice analogs have column densities above one hundred monolayers ( $1 \mathrm{ML}$ is commonly defined as $10^{15}$ molecules $/ \mathrm{cm}^{2}$ ), which are well above the canonical thickness of a thin interstellar ice mantle ( 40 ML; Bisschop et al. 2006). Therefore, these experiments more faithfully reproduce the thermal annealing of thicker ice mantles formed in cold circumstellar regions by grain agglomeration (and even cometary ices, see Sect. 4). Pre-cometary ice analog composition in experiments E10 and E11 is similar to that found in most interstellar and pre-cometary icy environments (Mumma \& Charnley 2011, and references therein). We also performed additional experiments (E6-E9) involving ices with a different composition to study in depth some particular effects. Experiments with pure ices of each component were performed as references to study mixture effects (E1-E5).

\section{Experimental results and discussion}

\subsection{Mixture effects on TPD curves of a pre-cometary ice analog}

Figure 1a shows the TPD curves of the pure ices (E1-E5), which are measured with the QMS of ISAC. The ices were deposited at $8 \mathrm{~K}$ and subsequently warmed up with a heating rate of $1 \mathrm{~K} / \mathrm{min}$. All curves are similar to those reported in previous works (e.g., Collings et al. 2004) with a broad feature that peaks at a different temperature according to the sublimation energy or the volatility of the species. The bumps that appear in these broad features are due to phase changes occurring in the ice between different amorphous structures, or from an amorphous to a crystalline
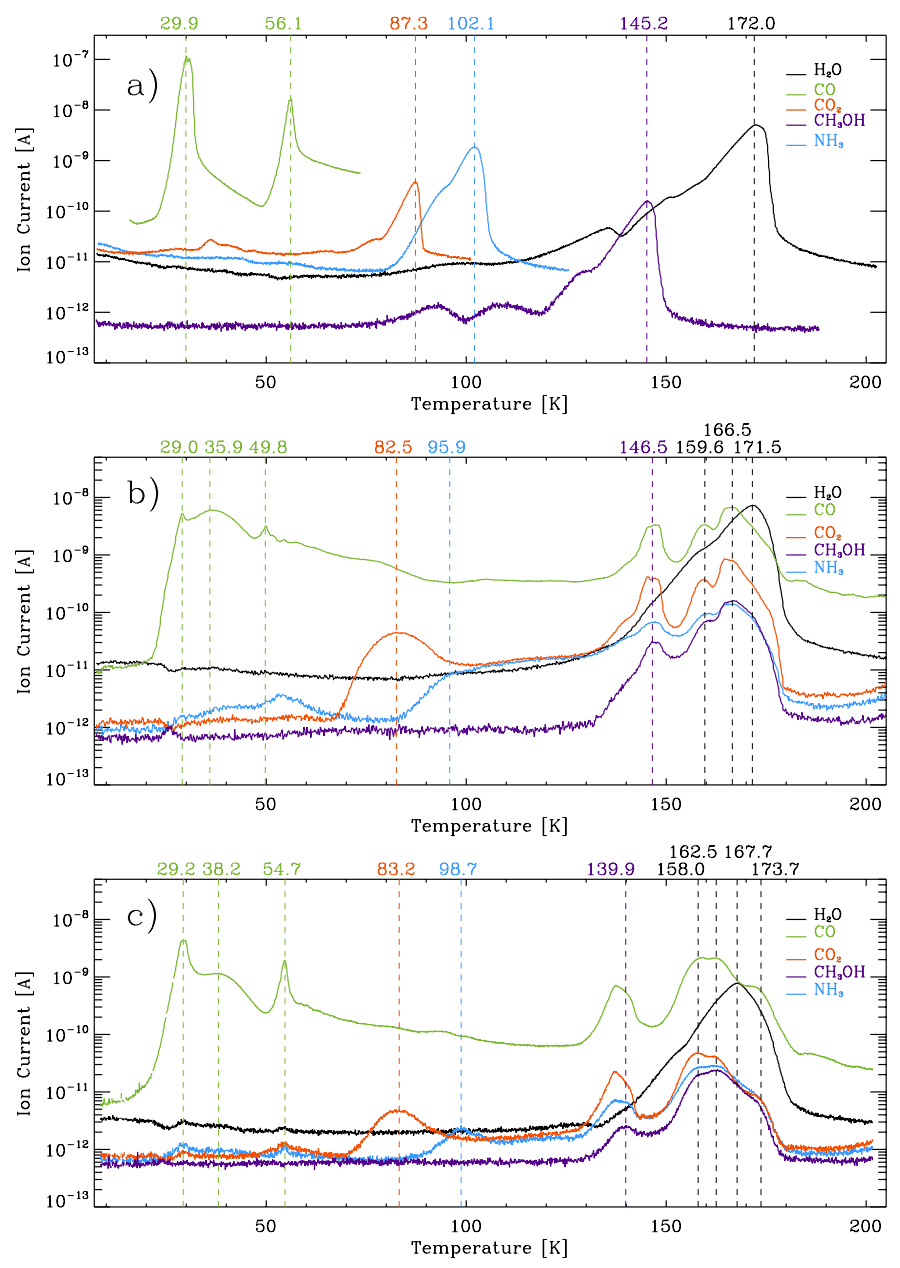

Fig. 1. TPD curves of each component in experiments a) E1-E5 (pure ices); b) E10 (pre-cometary ice mixture with heating rate $=2 \mathrm{~K} / \mathrm{min}$ ). Desorption of a molecule with mass fragment $\mathrm{m} / \mathrm{z}=15$ at $T<60 \mathrm{~K}$ does not correspond to $\mathrm{NH}_{3}$, since that feature is not shared with the $m / z=17$ fragment (not shown for clarity), and it may be due to a contaminant; c) E11 (pre-cometary ice mixture with heating rate = $1 \mathrm{~K} / \mathrm{min}$ ). Temperatures of desorption peaks are indicated. Ion current in the $y$-axis corresponds approximately to the partial pressure (mbar) in the main chamber. Note that the $y$-axis is on a logarithmic scale.

phase. In all cases the previous structure has a higher vapor pressure. Therefore, the desorption rate first increases with temperature, then decreases as the phase change occurs, and increases again after it is completed. These bumps are more easily observed for thick ices (Brown et al. 2006).

Unlike the rest of the studied molecules, CO molecules bind more strongly to the $\mathrm{KBr}$ substrate than to themselves. Therefore, its curve presents two peaks: the first peak at $T=$ $29.9 \mathrm{~K}$ corresponds to the bulk (multilayer) desorption, while the second peak at $T=56.1 \mathrm{~K}$ corresponds to molecules desorbing directly from the substrate (monolayer desorption).

Figure $1 \mathrm{~b}$ shows the TPD curve of each ice component in a pre-cometary ice mixture co-deposited at $8 \mathrm{~K}$ and warmed up with a heating rate of $2 \mathrm{~K} / \mathrm{min}$ (experiment E10). All species show desorption peaks at temperatures near the ones corresponding to pure ices. Desorption of $\mathrm{NH}_{3}$ and $\mathrm{CH}_{3} \mathrm{OH}$ at temperatures compatible with pure desorption was not previously documented for co-deposited mixtures (Collings et al. 2004; Brown et al. 2006). Desorption of molecules from a pure ice environment is considered evidence of segregation occuring in the ice mixture, 
at least to some extent (Bisschop et al. 2006). Segregation of species during thermal annealing is studied in more depth in Sect. 3.5.

We detect the two desorption peaks of $\mathrm{CO}$ between $30 \mathrm{~K}$ and $50 \mathrm{~K}$, as reported in Collings et al. (2004) for a co-deposited binary mixture, and also a third peak at a temperature below $30 \mathrm{~K}$ thanks to a better sensitivity of the QMS and a slower heating rate. The peak detected at $29.0 \mathrm{~K}$ and the peak at $49.8 \mathrm{~K}$ correspond to the multilayer and monolayer desorption of $\mathrm{CO}$ ice, respectively, which are also observed in the pure ice experiment (E2, Fig. 1a). The origin of the peak at $35.9 \mathrm{~K}$, attributed by Collings et al. (2004) to monolayer desorption, is not fully understood.

In addition, $\mathrm{CO}$ molecules in the ice keep desorbing continuously at temperatures higher than those corresponding to multilayer and monolayer desorptions, according to the QMS data, see Fig. 1a. This was confirmed by means of IR spectroscopy. Figure 4 shows the evolution of the $\mathrm{C}=\mathrm{O}$ stretching band at $2139 \mathrm{~cm}^{-1}$ during warm-up of the pre-cometary ice mixture in experiment E10. The absorbance decreases between the monolayer desorption at $T \sim 50 \mathrm{~K}$ and the next desorption peak at $T \sim 146 \mathrm{~K}$. Assuming that the band strength does not change very significantly with temperature, as it is the case for pure CO ices, the column density drops from $\sim 81 \%$ of its initial value at $T \sim 63 \mathrm{~K}$ to $\sim 48 \%$ at $T \sim 133 \mathrm{~K}$.

All the species except $\mathrm{H}_{2} \mathrm{O}$ present desorption peaks at temperatures higher than the corresponding temperature for pure ices. This indicates that these molecules are retained in the water ice structure (Bar-Nun et al. 1985; Collings et al. 2004; Fayolle et al. 2011). A fraction of the trapped molecules is released in the "volcano" desorption (Smith et al. 1997) when the change from amorphous to cubic crystalline water ice occurs at $T \sim 160 \mathrm{~K}$. Volcano desorption of $\mathrm{NH}_{3}$ molecules in co-deposited mixtures with water was not reported in Collings et al. (2004), while volcano desorption of $\mathrm{CH}_{3} \mathrm{OH}$ had already been detected by Brown et al. (2006). Another fraction of the trapped molecules co-desorbs later with water, which peaks at $T \sim 171 \mathrm{~K}$.

A second volcano desorption in the 160-170 K temperature range, corresponding to the release of molecules during the phase change from cubic to hexagonal water ice, is probably responsible for the shift between the co-desorption peaks temperature $(T \sim 166 \mathrm{~K})$ and the water desorption peak temperature. This transformation takes place at $213 \mathrm{~K}$ in pure ices (Dowell $\&$ Rinfret 1960), but the presence of large quantities of trapped molecules eases the phase change at a lower temperature range (Notesco \& Bar-Nun 2000). Therefore, we found that

- No shift is detected when the concentration of $\mathrm{H}_{2} \mathrm{O}$ is above $85 \%$ (E6, Fig. 2a; E8, Fig. 2d).

- A shift of $\sim 5 \mathrm{~K}$ is observed when the concentration is $\sim 70 \%$ (E7, Fig. 2b; E10, Fig. 1b; E11 Fig. 1c).

- A shift of $\sim 10 \mathrm{~K}$ is observed when the concentration is below $60 \%$ (E9, Fig. 2e).

The three types of desorption described so far (desorption as for the pure ice, volcano desorption and co-desorption with water) are common to most of the binary mixtures with $\mathrm{H}_{2} \mathrm{O}$. In a first approximation, the thermal desorption behavior of interstellar and cometary ices is dominated by interactions with the water ice matrix (diffusion and entrapment of molecules within the water ice structure). However, co-desorption of the three most volatile species $\left(\mathrm{CO}, \mathrm{CO}_{2}\right.$, and $\left.\mathrm{NH}_{3}\right)$ with $\mathrm{CH}_{3} \mathrm{OH}$ at $T \sim 146 \mathrm{~K}$ is also observed in three of the studied multicomponent mixtures, where desorption of methanol at a temperature near the one corresponding to the pure ice is detected (E10, Fig. 1b; E11,

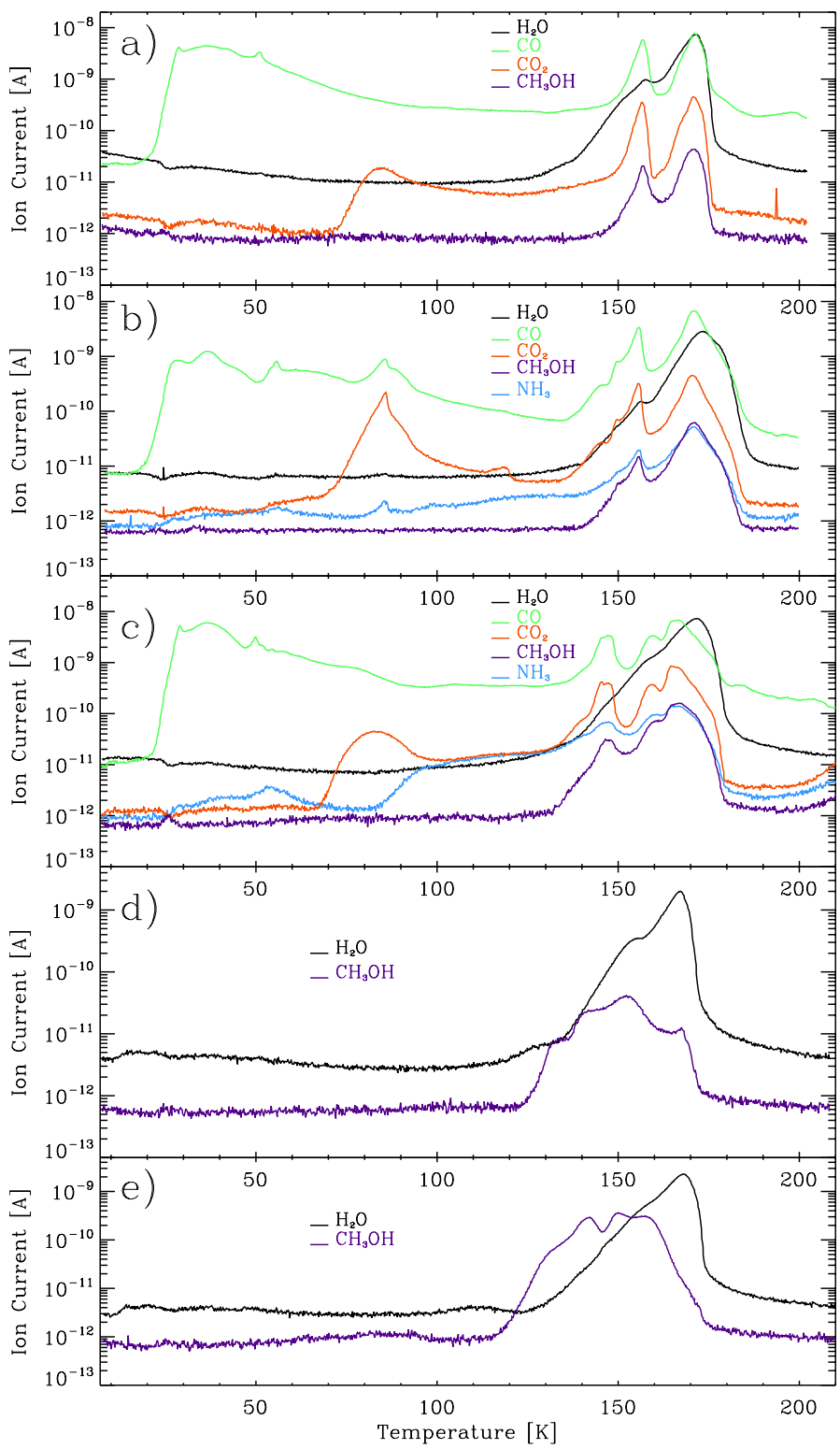

Fig. 2. TPD curves of each component in experiments a) E6; b) E7; c) $\mathrm{E} 10$; d) $\mathrm{E} 8$; and e) $\mathrm{E} 9$. The concentration of $\mathrm{CH}_{3} \mathrm{OH}$ in the mixture increases from a) to e). Ion current in the $y$-axis corresponds approximately to the partial pressure (mbar) in the main chamber. Note that the $y$-axis is on a logarithmic scale.

Fig. 1c; and E7, Fig. 2b). These co-desorption peaks cannot be reproduced in binary ice mixtures with water.

\subsubsection{Effects of the heating rate}

Figure 1c shows the TPD curves of a pre-cometary ice mixture with a heating rate of $1 \mathrm{~K} / \mathrm{min}$ (E11). The curves are rather similar to those observed in experiment E10 (Fig. 1b) with a similar ice composition and thickness and a heating rate two times faster ( $2 \mathrm{~K} / \mathrm{min}$ ). All desorption peaks observed in Fig. $1 \mathrm{~b}$ are detected in Fig. 1c with the addition of a second co-desorption bump at $T \sim 174 \mathrm{~K}$. Temperatures of most of the desorption peaks are cooler in experiment E11 (see Table 3), as it is expected for a slower heating rate. It is unclear, though, why monolayer desorption of $\mathrm{CO}$ takes place at higher temperatures. 
Table 3. Temperature of desorption peaks in experiments E11 (Fig. 1c, heating rate $=1 \mathrm{~K} / \mathrm{min}$ ) and $\mathrm{E} 10$ (Fig. $1 \mathrm{~b}$, heating rate $=2 \mathrm{~K} / \mathrm{min}$ ).

\begin{tabular}{ccc}
\hline \hline \multirow{2}{*}{$\begin{array}{c}\text { Desorption } \\
\text { peak }\end{array}$} & \multicolumn{2}{c}{ Temperature $(\mathrm{K})$} \\
\cline { 2 - 3 } & $\mathrm{E} 11$ & $\mathrm{E} 10$ \\
\hline $\mathrm{CO}$ multilayer & 29.2 & 29.0 \\
\hline $\mathrm{CO}$ & 38.2 & 35.9 \\
monolayer & 54.7 & 49.8 \\
\hline $\mathrm{CO}_{2}$ & 83.2 & 82.5 \\
\hline $\mathrm{NH}_{3}$ & 98.7 & $\sim 95.9$ \\
\hline $\mathrm{CH}_{3} \mathrm{OH}$ & 140.0 & 146.5 \\
\hline $\mathrm{volcano}$ & 158.0 & 159.6 \\
\hline co-desorption $/ \mathrm{H}_{2} \mathrm{O}$ & 162.5 & 166.5 \\
\hline $\mathrm{H}_{2} \mathrm{O}$ & 167.7 & 171.5 \\
\hline
\end{tabular}

Notes. Temperature of the ammonia desorption peak in E10 (Fig. 1b) is not well constrained due to the shape of the curve. Temperatures of volcano and co-desorption peaks are approximate, since they do not coincide exactly for all the species.

\subsection{Binding energies of pure ices}

The desorption rate of pure ices is given by the Polanyi-Wigner equation,

$\frac{\mathrm{d} N_{\mathrm{g}}}{\mathrm{d} t}=v_{i} N_{\mathrm{s}}^{i} \exp \left(-E_{\mathrm{des}} / T\right)$

where $\mathrm{d} N_{\mathrm{g}} / \mathrm{d} t$ is the desorption rate in molecules $\mathrm{cm}^{-2} \mathrm{~s}^{-1}, v_{i}$ a pre-exponential constant for order $i$ in molecules ${ }^{1-i} \mathrm{~cm}^{-2(1-i)} \mathrm{s}^{-1}$, $N_{\mathrm{s}}$ the column density of the desorbing ice at time $t$ in molecules $\mathrm{cm}^{-2}, E_{\mathrm{des}}$ the binding energy in $\mathrm{K}$, and $T$ the ice temperature in $\mathrm{K}$ at time $t$. Both $E_{\mathrm{des}}$ and $v_{i}$ depend, in principle, on the ice thickness, but this dependence is not thought to be large, even for non-zero desorption orders (Bisschop et al. 2006). However, Bolina et al. (2005a) reported a difference of $\sim 20 \%$ in the binding energy of $\mathrm{CH}_{3} \mathrm{OH}$ using ices with a range of thicknesses spanning one order of magnitude and a non-zero desorption order.

Desorption peaks of pure ices can thus be fitted using the Polanyi-Wigner expression and the relation,

$\frac{\mathrm{d} N_{\mathrm{g}}}{\mathrm{d} t}=\frac{\mathrm{d} N_{\mathrm{g}}}{\mathrm{d} T} \frac{\mathrm{d} T}{\mathrm{~d} t}$

where $\mathrm{d} T / \mathrm{d} t$ is the heating rate.

Table 3 presents an estimate of the pre-exponential constant and the binding energy for the five pure ices, assuming zeroth order kinetics, which is a reasonable approximation for multilayer desorption peaks (Noble et al. 2012). We used a $\chi^{2}$ parameter to find the best fit to the experimental desorption peaks. Since this parameter is strongly dependent on the range of temperatures for which the fit is done, we have used the binding energy estimated by the TPD peak position with Eq. (4) (Attard \& Barnes 1998) as a reference.

$E_{\mathrm{des}}=T_{\mathrm{des}} \times 30.068$.

Errors indicated in Table 3 arise from the range of values leading to a similar $\chi^{2}$. The pre-exponential constant found for the $\mathrm{CO}$ desorption peak is one order of magnitude larger than the values previously reported, while the binding energy coincides within errors (Muñoz Caro et al. 2010; Noble et al. 2012). The binding energy obtained for the $\mathrm{CO}_{2}$ desorption peak also coincides within errors with the value reported by Sandford \& Allamandola (1990), although it is $~ 10 \%$ larger than
Table 4. Desorption rate parameters for the five pure ices.

\begin{tabular}{cccc}
\hline \hline Species & $\begin{array}{c}v_{0} / 10^{27} \\
\left(\mathrm{~mol} \mathrm{~cm}^{-2} \mathrm{~s}^{-1}\right)\end{array}$ & $\begin{array}{c}E_{\text {des }} \\
(\mathrm{K})\end{array}$ & $\begin{array}{c}\text { Estimated } \\
E_{\text {des }}(\mathrm{K})\end{array}$ \\
\hline $\mathrm{CO}$ & $6.5_{-4.6}^{+15.3}$ & $890 \pm 35$ & 899 \\
$\mathrm{CO}_{2}$ & $5.2_{-1.6}^{+4.3}$ & $2605_{-30}^{+50}$ & 2625 \\
$\mathrm{NH}_{3}$ & $2.1_{-1.2}^{+2.7}$ & $2965 \pm 80$ & 3070 \\
$\mathrm{CH}_{3} \mathrm{OH}$ & $5.3_{-2.7}^{+16.0}$ & $4355_{-100}^{+200}$ & 4366 \\
$\mathrm{H}_{2} \mathrm{O}$ & $32.1_{-8.5}^{+12.8}$ & $5165 \pm 55$ & 5172 \\
\hline
\end{tabular}

Notes. Columns 2 and 3: Pre-exponential constants and binding energies assuming the Polanyi-Wigner desorption rate equation and zeroth order kinetics. Errors arise from the range of values leading to a similar $\chi^{2}$. Column 4: estimated binding energy from Eq. (4).

that reported by Noble et al. (2012) while the pre-exponential constant is a factor $\sim 5$ larger in our case. Bolina et al. (2005b) used a desorption order of $0.25 \pm 0.05$ for the $\mathrm{NH}_{3}$ desorption peak leading to a binding energy close to the one reported here, but a pre-exponential constant two orders of magnitude lower. In the case of $\mathrm{CH}_{3} \mathrm{OH}$, the binding energy in Table 3 is within the range of values reported by Bolina et al. (2005a), where a desorption order of $0.35 \pm 0.21$ is used. Pre-exponential constant for $\mathrm{H}_{2} \mathrm{O}$ desorption coincides within errors with that reported in Fraser et al. (2001), although the binding energy we obtained is $\sim 10 \%$ lower.

\subsection{Entrapment of molecules in multicomponent ices}

Since ice diffusion barriers are proportional to the volatility of the species (Öberg et al. 2009), volatile molecules like CO or, to a lesser extent, $\mathrm{CO}_{2}$, are able to diffuse more easily through the amorphous solid water. Therefore, multilayer and/or monolayer desorption peaks are usually detected for these two species. In exchange, less volatile components, like $\mathrm{NH}_{3}$ and $\mathrm{CH}_{3} \mathrm{OH}$, do not diffuse so easily, and are retained to a larger extent within the water ice structure. Desorption of $\mathrm{NH}_{3}$ and $\mathrm{CH}_{3} \mathrm{OH}$ prior to the volcano desorption had not been previously detected in co-deposited mixtures (Collings et al. 2004; Brown et al. 2006). This might be due to a lower sensitivity of the mass spectrometers used in previous works and the faster heating rate applied, which hinders the detection of these desorptions. These peaks have been detected in our pre-cometary ice mixtures (Figs. 1b and c) with similar mixture ratios and thicker ices.

We have studied the effect of the concentration on the entrapment of $\mathrm{NH}_{3}$ and $\mathrm{CH}_{3} \mathrm{OH}$ molecules (experiments E6-E10, Fig. 2a-e) using ice analogs with a different composition than that of the pre-cometary ice mixtures. The fraction of trapped molecules can be inferred from the relative intensities of the peaks corresponding to pure desorption and the volcano and co-desorption peaks, which correspond to desorption of trapped molecules. As it has been reported for other species, this fraction decreases with an increase of the species concentration relative to water:

- Below a concentration of $\sim 2 \%$ relative to water, desorption of $\mathrm{CH}_{3} \mathrm{OH}$, expected at $T \sim 145 \mathrm{~K}$ according to Fig. 1a, was not detected (E6, Fig. 2a), and only volcano and co-desorption peaks were observed. Therefore, all $\mathrm{CH}_{3} \mathrm{OH}$ molecules were trapped in the water ice structure.

- With a concentration of $\sim 3 \%$, desorption of $\mathrm{CH}_{3} \mathrm{OH}$ at $T \sim$ $149 \mathrm{~K}$, prior to the volcano and co-desorption peaks, was clearly detected thanks to the co-desorption of $\mathrm{CO}$ and $\mathrm{CO}_{2}$ 


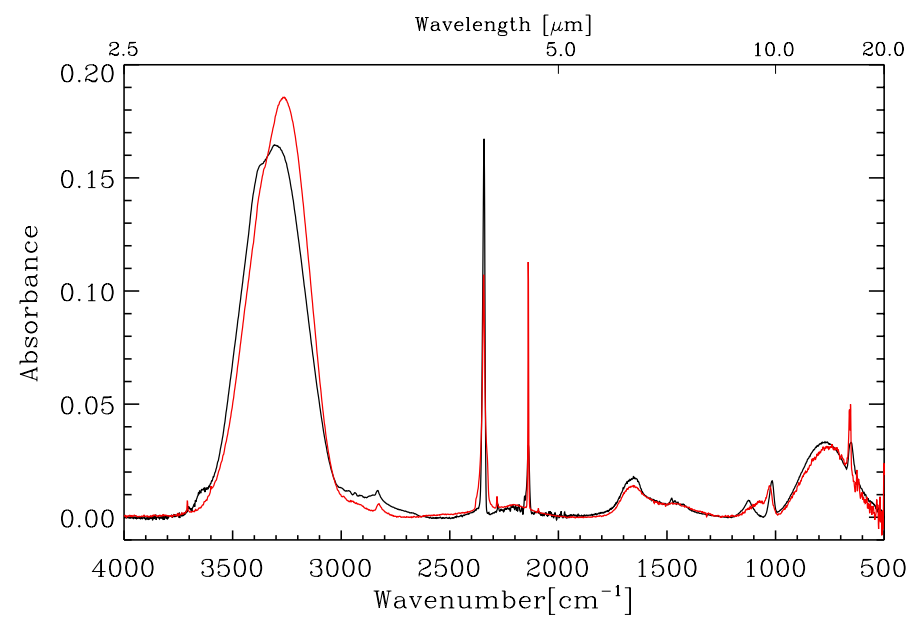

Fig. 3. Black: IR spectra of a pre-cometary ice mixture (E11) registered at $8 \mathrm{~K}$. Red: Sum of the IR spectra of individual pure ice components (E1-E5) registered at $8 \mathrm{~K}$; they were multiplied by a factor to reproduce the composition of the pre-cometary ice mixture.

(E7, Fig. 2b)). The partial pressure of $\mathrm{NH}_{3}$ in the chamber also increases slightly in the temperature range at which pure desorption of this species is expected.

- Above a concentration of $\sim 5 \%$, desorption peaks equivalent to those of pure $\mathrm{NH}_{3}$ and $\mathrm{CH}_{3} \mathrm{OH}$, respectively, at $95.9 \mathrm{~K}$ and $146.5 \mathrm{~K}$ are clearly identified (E10, Fig. 2c).

- The intensity of the desorption peak equivalent to pure $\mathrm{CH}_{3} \mathrm{OH}$ increases with its concentration in the waterdominated ice matrix (E8, Fig. 2d; E9, Fig. 2e).

Clear trends are observed regardless of the total column density deposited in each experiment, which indicates that the total thickness of the ice is not as relevant for ices of a few hundred monolayers as it is for thin ices.

\subsection{Mixture effects on IR spectra of a pre-cometary ice analog}

The IR spectra collected during the heating of the ice analogs compliment the analysis of the TPD data. Figure 3 shows the IR spectrum at $8 \mathrm{~K}$ of the pre-cometary ice mixture in experiment E11, along with a spectral fit consisting of the sum of the individual pure ice spectra (E1-E5) that are also registered at $8 \mathrm{~K}$, after scaling with a factor to reproduce the composition of the ice mixture.

The IR spectrum of the pre-cometary ice mixture appears to be fairly well reproduced by the sum of individual spectra of pure ices, although some significant differences are observed, which are discussed below in order of decreasing wavenumber. Most of these differences are due to interactions between water molecules and the rest of ice components and have been previously reported for binary mixtures. This is in line with the similarity between TPD curves in pre-cometary ice mixtures and in binary mixtures with the exception for the co-desorption of $\mathrm{CO}$, $\mathrm{CO}_{2}$, and $\mathrm{NH}_{3}$ with $\mathrm{CH}_{3} \mathrm{OH}$, as mentioned in Sect. 3.1.

There is a new feature at $\sim 3650 \mathrm{~cm}^{-1}$ which is not reproduced by the sum of pure ice spectra. This feature arises from the interactions of $\mathrm{CO}$ and $\mathrm{CO}_{2}$ molecules with the amorphous solid water (Hagen et al. 1983). Hydrogen bonds formed between $\mathrm{CO}_{2}$ and $\mathrm{H}_{2} \mathrm{O}$ molecules are weaker than those formed between water molecules. The molecule $\mathrm{CO}$ is not even able to form these bonds. Therefore, there are some water $\mathrm{OH}$-groups,

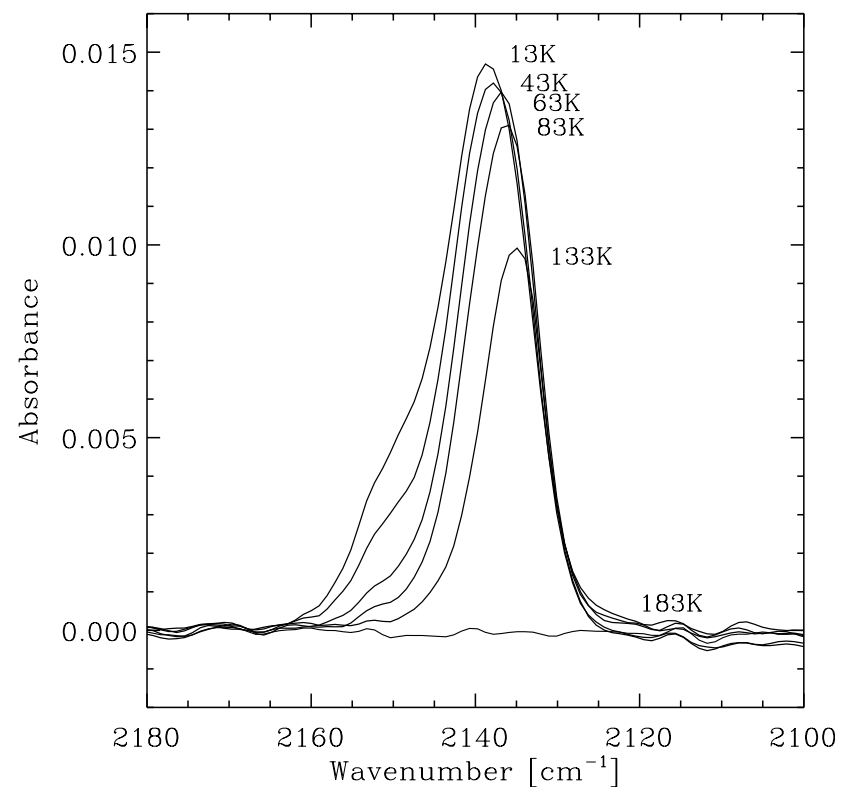

Fig. 4. Evolution of the $\mathrm{C}=\mathrm{O}$ stretching band of $\mathrm{CO}$ during the warm-up of a pre-cometary ice mixture (experiment E10).

who are not connected to the hydrogen bonded network and whose $\mathrm{O}-\mathrm{H}$ stretching mode has a higher frequency, leading to the appearance of this feature in the blue side of the $\mathrm{O}-\mathrm{H}$ stretching band.

Moreover, the extent of intermolecular coupling in the water ice structure becomes smaller and the hydrogen bonds that do form become weaker, which explains the blueshift of the $\mathrm{O}-\mathrm{H}$ stretching band of $\mathrm{H}_{2} \mathrm{O}$, from $3262 \mathrm{~cm}^{-1}$ to $3307 \mathrm{~cm}^{-1}$ due to the presence of $\mathrm{CO}$ and $\mathrm{CO}_{2}$. In addition, this band has a lower intensity than expected, since the induced polarization is reduced, but has a more intense low-frequency wing due to the presence of $\mathrm{CH}_{3} \mathrm{OH}$ and $\mathrm{NH}_{3}$ in the ice mixture (Hagen et al. 1983).

The band strength of the $\mathrm{N}-\mathrm{H}$ stretching mode of $\mathrm{NH}_{3}$ seems to be also enhanced in the mixture, since the shoulder at $\sim 3370 \mathrm{~cm}^{-1}$ is more intense than expected. Due to the overlap with the $\mathrm{O}-\mathrm{H}$ stretching mode of $\mathrm{H}_{2} \mathrm{O}$, this mixture effect is very difficult to quantify (d'Hendecourt \& Allamandola 1986).

The $\mathrm{C}=\mathrm{O}$ stretching band of $\mathrm{CO}_{2}$ at $2344 \mathrm{~cm}^{-1}$ is slightly redshifted to $2342 \mathrm{~cm}^{-1}$ (see left panel of Fig. 5), and it is sharper in the mixture. The behavior of this band in mixtures with $\mathrm{H}_{2} \mathrm{O}$ depends on the concentration of $\mathrm{CO}_{2}$ and also on the presence of other polar molecules, such as $\mathrm{CH}_{3} \mathrm{OH}$ (Sandford \& Allamandola 1990; Ehrenfreund et al. 1999).

The $\mathrm{C}=\mathrm{O}$ stretching band of $\mathrm{CO}$ at $2139 \mathrm{~cm}^{-1}$ has a shoulder on its blue side, peaking at $2152 \mathrm{~cm}^{-1}$ (see Fig. 4). This shoulder is believed to originate from the interaction of $\mathrm{CO}$ molecules with dangling $\mathrm{O}-\mathrm{H}$ bonds of water molecules at a surface or discontinuity that cannot complete a tetrahedrally hydrogen bonded network (Collings et al. 2003a). Surprisingly, this peak is not observed in astronomical spectra, which can be reproduced with a mixture of solid $\mathrm{CO}$ and $\mathrm{CH}_{3} \mathrm{OH}$ without the presence of $\mathrm{H}_{2} \mathrm{O}$ (Cuppen et al. 2011).

The band strength of the $\mathrm{O}-\mathrm{H}$ bending mode at $\sim 1650 \mathrm{~cm}^{-1}$ is increased in the mixture due to the presence of $\mathrm{CO}$ and $\mathrm{CO}_{2}$ (Hagen et al. 1983).

The umbrella mode of $\mathrm{NH}_{3}$ at $1067 \mathrm{~cm}^{-1}$ is blueshifted to $1124 \mathrm{~cm}^{-1}$ in the mixture. This is observed in binary mixtures with $\mathrm{H}_{2} \mathrm{O}$ (Hagen et al. 1983; d'Hendecourt \& Allamandola 1986). The relatively strong shift of this band manifests the intermolecular bond strength between $\mathrm{NH}_{3}$ and $\mathrm{H}_{2} \mathrm{O}$ molecules. 


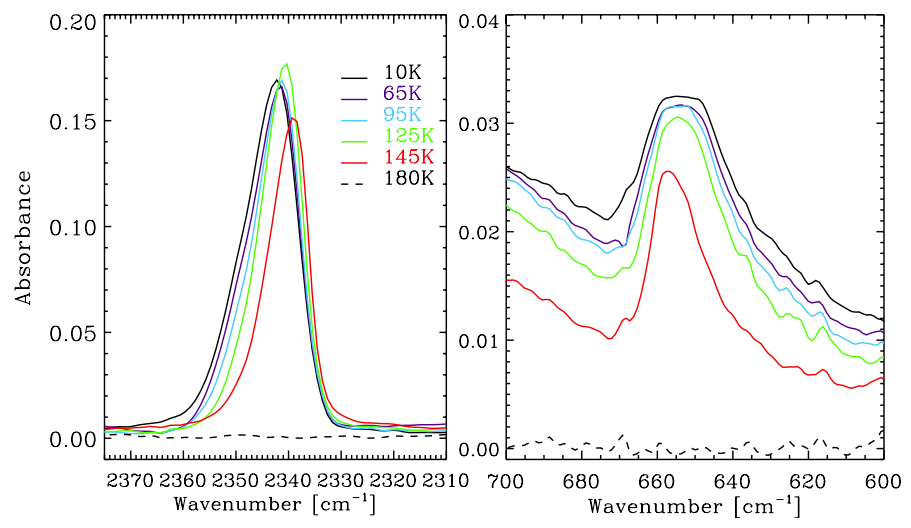

Fig. 5. Evolution of the IR bands of $\mathrm{CO}_{2}$ during the warm-up of a precometary ice mixture (experiment E11). Left: $\mathrm{C}=\mathrm{O}$ stretching mode at $\sim 2342 \mathrm{~cm}^{-1}$. Right: bending mode at $\sim 655 \mathrm{~cm}^{-1}$.

On the other hand, the $\mathrm{C}-\mathrm{O}$ stretching band of $\mathrm{CH}_{3} \mathrm{OH}$ is redshifted from $1025 \mathrm{~cm}^{-1}$ to $1016 \mathrm{~cm}^{-1}$. This effect has been also observed in binary mixtures (d'Hendecourt \& Allamandola 1986). This relatively small shift indicates that the hydrogen bond strength between $\mathrm{CH}_{3} \mathrm{OH}$ and $\mathrm{H}_{2} \mathrm{O}$ molecules in the ice, and the $\mathrm{CH}_{3} \mathrm{OH}-\mathrm{CH}_{3} \mathrm{OH}$ bonds are only slightly different. This observation is confirmed by the similar binding energies inferred from the desorption temperatures.

The degenerate bending modes of $\mathrm{CO}_{2}$ at $\sim 655 \mathrm{~cm}^{-1}$ lose the double-peak structure in the mixture, as previously reported for binary mixtures with water (Sandford \& Allamandola 1990).

\subsection{Evolution of IR spectra during thermal annealing}

Figure 4 shows the gradual disappearance of the shoulder in the blue side of the $\mathrm{C}=\mathrm{O}$ stretching band of $\mathrm{CO}$ during the warmup. It is negligible at $\sim 63 \mathrm{~K}$ and has completely disappeared at $\sim 83 \mathrm{~K}$. This phenomenon is thought to be an effect of the decrease in the number of dangling $\mathrm{O}-\mathrm{H}$ bonds in the water ice structure during the transition from the highly porous to the less porous amorphous solid water (Sandford et al. 1988; Collings et al. 2003b). Öberg et al. (2009) observed an increase in the intensity of the main peak at $2138 \mathrm{~cm}^{-1}$ relative to the shoulder at $T \sim 25 \mathrm{~K}$ (before the onset of the phase transition), suggesting that the change in the IR band is due to segregation of $\mathrm{CO}$ in the ice. Since the loss of the shoulder in Fig. 4 takes place along with a redshift of the main peak, which is characteristic of binary mixtures with water (Sandford et al. 1988) in our experiment these effects are most likely due to structural changes in the ice. Therefore, no strong evidence of CO segregation is found in the IR spectra, despite the detection of desorption peaks at temperatures corresponding to pure CO ice in the TPD curves. This probably means that $\mathrm{CO}$ segregation takes place only to a small extent (to a small spatial extent within the ice and/or in a short time span before multilayer desorption occurs) and its effects cannot be detected with the FTIR spectrometer because it is less sensitive than the QMS.

The evolution of the $\mathrm{CO}_{2}$ stretching and bending bands is shown in the left and right panel of Fig. 5 , respectively. The $\mathrm{C}=\mathrm{O}$ stretching band at $2342 \mathrm{~cm}^{-1}$ slightly increases its intensity between $65 \mathrm{~K}$ and $125 \mathrm{~K}$ at the same temperature range in which pure $\mathrm{CO}_{2}$ desorption takes place. This behavior is shared with the $\mathrm{C}=\mathrm{O}$ stretching band of pure $\mathrm{CO}_{2}$ ice, but we do not observe other effects of ice segregation, as reported by Öberg et al. (2009) for a binary mixture of $\mathrm{CO}_{2}$ and $\mathrm{H}_{2} \mathrm{O}$, such as the blueshift or the
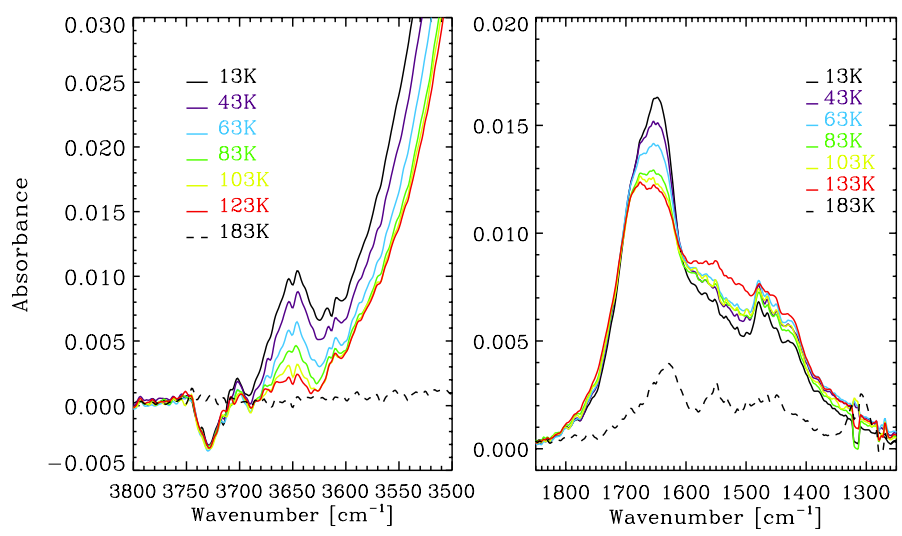

Fig. 6. Evolution of the IR bands of $\mathrm{H}_{2} \mathrm{O}$ during the warm-up of a precometary ice mixture (experiment E10). Left: O-H stretching mode at $\sim 3650 \mathrm{~cm}^{-1}$. Negative absorbances are due to the atmospheric compensation applied by the spectrometer software. Right: O-H bending mode at $\sim 1654 \mathrm{~cm}^{-1}$.

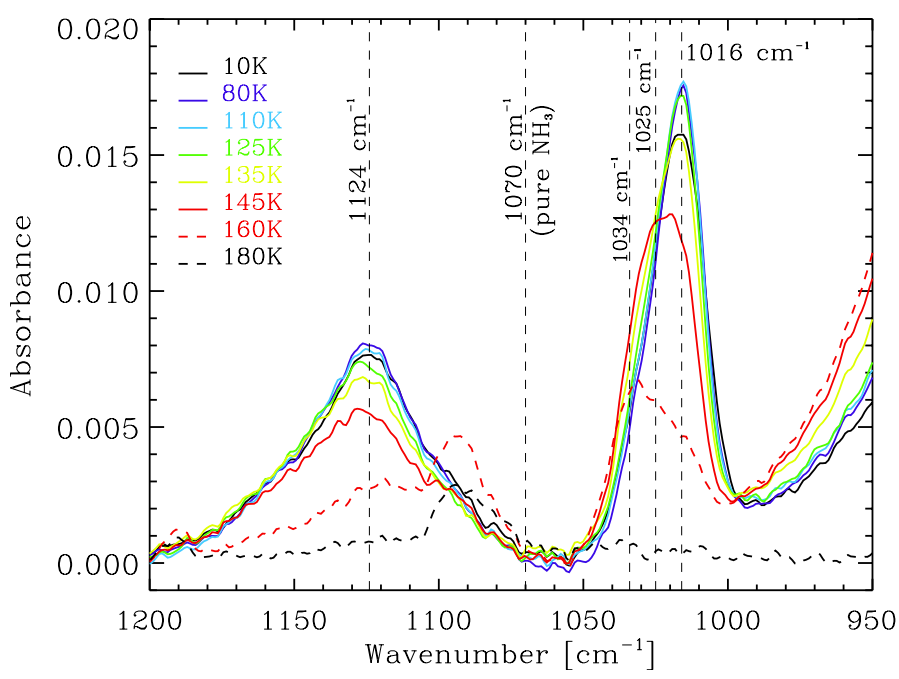

Fig. 7. Evolution of the umbrella mode of $\mathrm{NH}_{3}$ and the $\mathrm{C}-\mathrm{O}$ stretching mode of $\mathrm{CH}_{3} \mathrm{OH}$ during the warm-up of a pre-cometary ice mixture (experiment E11).

appearance of a new feature in the blue side of this band (Instead, the redshift increases to $2339 \mathrm{~cm}^{-1}$ at higher temperatures in our ice mixture.), or the double peak structure of the degenerate bending modes. Again, there is no strong evidence of $\mathrm{CO}_{2}$ segregation in our IR spectra, probably because it takes place only to a small extent, as it does in the case of $\mathrm{CO}$ molecules.

The feature at $\sim 3650 \mathrm{~cm}^{-1}$, which corresponds to the $\mathrm{O}-\mathrm{H}$ stretching mode of water molecules that are not completely connected to the hydrogen bonded network due to interactions with $\mathrm{CO}$ and $\mathrm{CO}_{2}$ molecules, gradually disappears during the warmup (left panel of Fig. 6). This is most likely an effect of transition to a more compact ice structure (Isokoski 2013), since there is no reliable evidence of $\mathrm{CO}$ and $\mathrm{CO}_{2}$ segregation (see above). The intensity of the bending mode of $\mathrm{H}_{2} \mathrm{O}$ decreases in the same temperature range (right panel of Fig. 6), which can also be explained by compaction.

Figure 7 shows the evolution of the umbrella mode of $\mathrm{NH}_{3}$ at $1124 \mathrm{~cm}^{-1}$ and the $\mathrm{C}-\mathrm{O}$ stretching mode of $\mathrm{CH}_{3} \mathrm{OH}$ at $1016 \mathrm{~cm}^{-1}$. No substantial changes are observed in the former, but the latter changes its band profile and peak frequency during the warm-up. Above a temperature of $T \sim 110 \mathrm{~K}$ before the onset of the desorption of $\mathrm{CH}_{3} \mathrm{OH}$, the peak shifts toward shorter wavelengths. At $T \sim 145 \mathrm{~K}$, the band is broader and peaks at a 


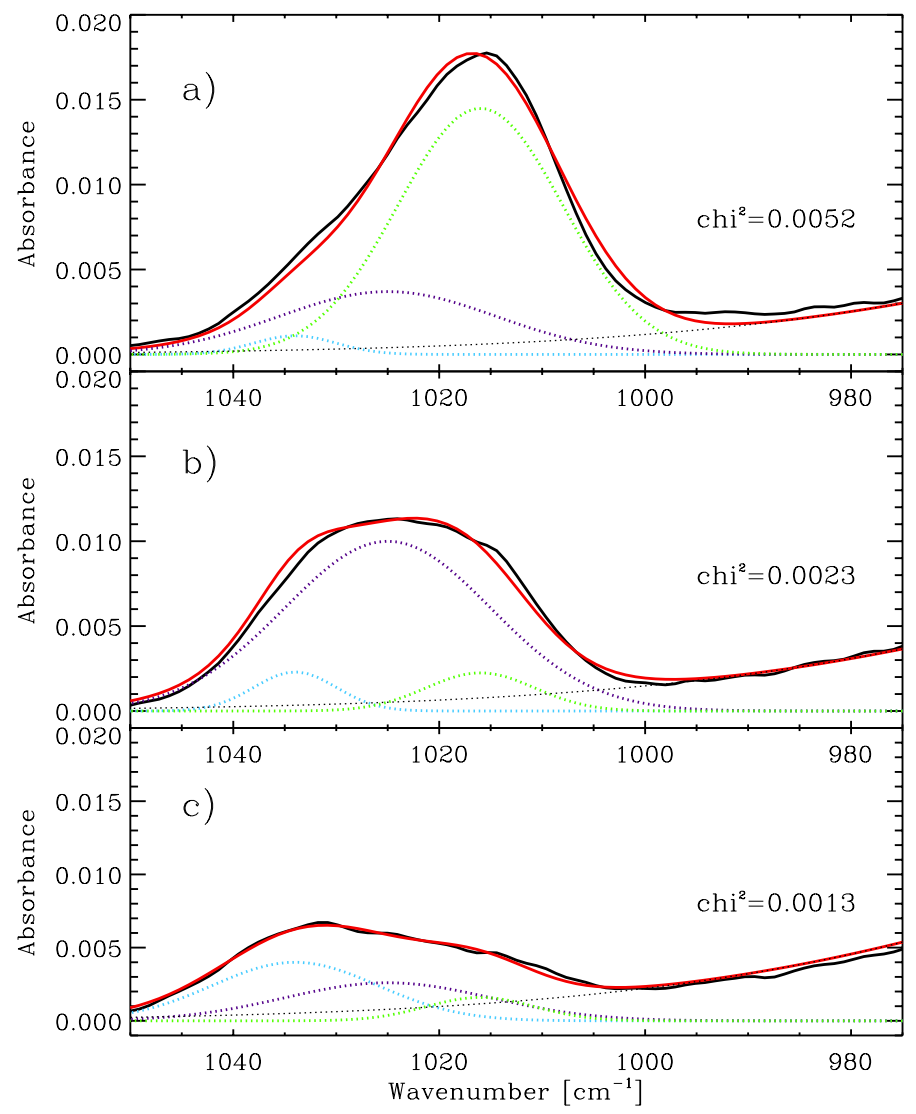

Fig. 8. $\mathrm{C}-\mathrm{O}$ stretching band of $\mathrm{CH}_{3} \mathrm{OH}$ (black) fitted with the sum of three Gaussians (red), which account for: mixed methanol (green dotted, centered at $1016 \mathrm{~cm}^{-1}$ ), segregated methanol (purple dotted, centered at $1025 \mathrm{~cm}^{-1}$ ), and methanol forming a type II clathrate hydrate with water molecules (blue dotted, centered at $1034 \mathrm{~cm}^{-1}$ ). A fourth Gaussian has been used to take into account the contribution of the blue wing of the $\mathrm{H}_{2} \mathrm{O}$ librational band (black dotted). The IR spectra correspond to a pre-cometary ice analog (E11) at a) $T=110 \mathrm{~K}$; b) $T=150 \mathrm{~K}$; and c) $T=160 \mathrm{~K}$. The $\chi^{2}$ parameter is indicated for each fit.

frequency close to that of pure $\mathrm{CH}_{3} \mathrm{OH}$ ice. At higher temperatures, the red component of the band gradually disappears and the peak is shifted to $\sim 1034 \mathrm{~cm}^{-1}$. This indicates that a fraction of the $\mathrm{CH}_{3} \mathrm{OH}$ molecules segregates in the ice at temperatures above $T \sim 110 \mathrm{~K}$, while other $\mathrm{CH}_{3} \mathrm{OH}$ molecules form a type II clathrate hydrate (Blake et al. 1991). Segregated molecules are able to desorb more easily than molecules in the clathrate hydrates, which mainly desorb at higher temperatures.

\subsection{1. $\mathrm{CH}_{3} \mathrm{OH}$ segregation}

The $\mathrm{CH}_{3} \mathrm{OH}$ molecules segregate enough to an extent to be detected by the FTIR spectrometer. We have fitted the $\mathrm{C}-\mathrm{O}$ stretching band of $\mathrm{CH}_{3} \mathrm{OH}$ in the IR spectra collected above $T \sim 110 \mathrm{~K}$ by using three Gaussians to estimate the contribution of the three types of methanol that can be found in the ice sample, namely: mixed methanol (centered at $1016 \mathrm{~cm}^{-1}$ ), segregated methanol (centered at $1025 \mathrm{~cm}^{-1}$ ), and methanol forming a type II clathrate hydrate with water molecules (centered at $1034 \mathrm{~cm}^{-1}$ ). A fourth Gaussian was used to take into account the contribution of the blue wing of the $\mathrm{H}_{2} \mathrm{O}$ librational band.

Figure 8 shows the fit for three spectra at $T \sim 110 \mathrm{~K}, 150 \mathrm{~K}$, and $160 \mathrm{~K}$, respectively. We used a $\chi^{2}$ parameter to find the best fit in each case. The evolution of the contribution of each

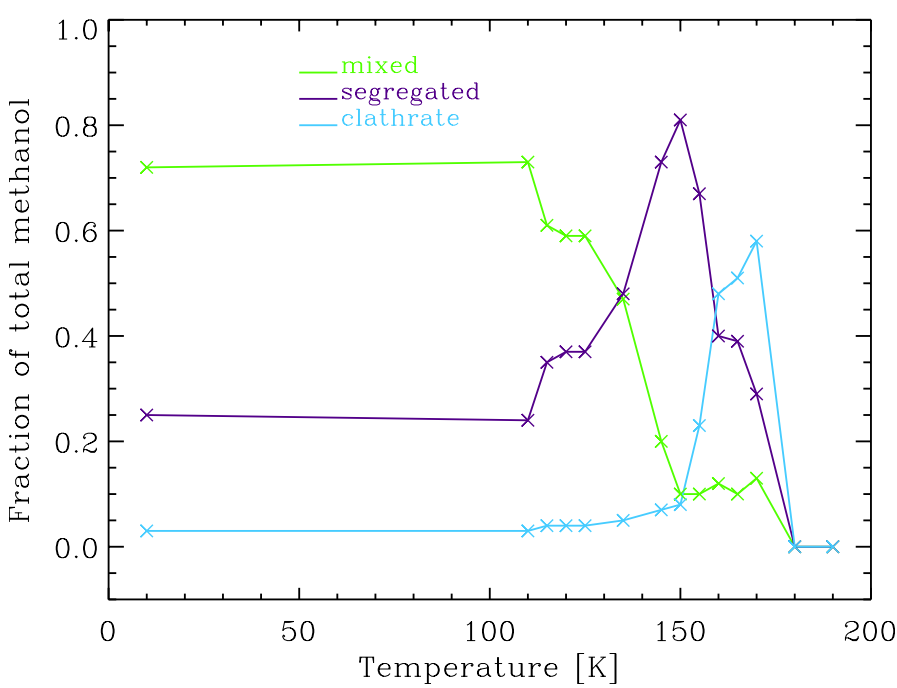

Fig. 9. Evolution of the contribution of mixed methanol (green), segregated methanol (purple), and methanol forming a type II clathrate hydrate (blue) to the total area of the $\mathrm{C}-\mathrm{O}$ stretching band of $\mathrm{CH}_{3} \mathrm{OH}$ during warm-up of a pre-cometary ice mixture (E11), according to fit with three Gaussians of the IR band. Temperatures for which the fit was done are indicated. Figure 8 shows three selected fits as an example.

Gaussian to the total area of the $\mathrm{C}-\mathrm{O}$ stretching band is shown in Fig. 9. Above $T \sim 110 \mathrm{~K}$, the contribution of segregated methanol and methanol in a type II clathrate hydrate to a lesser extent increases, while that of the mixed methanol decreases. At $T \sim 150 \mathrm{~K}$, before the volcano desorption occurs, segregated methanol dominates in the ice mixture and above that temperature methanol in a type II clathrate hydrate becomes the major contributor to the IR band until it is completely desorbed.

A final confirmation of the methanol segregation can be found in the $\mathrm{C}-\mathrm{H}$ stretching region of the spectrum. At these high temperatures, pure (segregated) $\mathrm{CH}_{3} \mathrm{OH}$ should be in its crystalline form. When pure $\mathrm{CH}_{3} \mathrm{OH}$ ice changes from the amorphous to the crystalline $\alpha$ structure, the asymmetric $\mathrm{C}-\mathrm{H}$ stretching mode (which at $8 \mathrm{~K}$ is a wide band peaking at $2930 \mathrm{~cm}^{-1}$ with a shoulder at $\sim 2984 \mathrm{~cm}^{-1}$ ) is perfectly resolved into the $a^{\prime}\left(v_{2}\right)$ and $a^{\prime \prime}\left(v_{9}\right)$ modes at $2984 \mathrm{~cm}^{-1}$ and $2856 \mathrm{~cm}^{-1}$, respectively, while the symmetric mode $\left(v_{3}\right)$ at $2827 \mathrm{~cm}^{-1}$ remains basically unchanged (Falk \& Whalley 1961). Unfortunately, the $v_{9}$ mode is barely detected in the pre-cometary ice mixture (Fig. 3), and it is not possible to confirm this process, but it is observed in a binary mixture with a higher concentration of methanol (experiment E9, Fig. 10). In this mixture, we observe three peaks at $2989 \mathrm{~cm}^{-1}, 2960 \mathrm{~cm}^{-1}$, and $2933 \mathrm{~cm}^{-1}$ at $8 \mathrm{~K}$, instead of the wide band peaking at $2930 \mathrm{~cm}^{-1}$. These peaks may arise from the interaction between $\mathrm{CH}_{3} \mathrm{OH}$ and $\mathrm{H}_{2} \mathrm{O}$. When the temperature raises to $T \sim 113 \mathrm{~K}$, these three peaks begin to change, and at $T \sim 143 \mathrm{~K}$, two peaks are clearly identified at frequencies near those of the $a^{\prime}$ and $a^{\prime \prime}$ asymmetric stretching modes $\left(2988 \mathrm{~cm}^{-1}\right.$ and $\left.2948 \mathrm{~cm}^{-1}\right)$. This proves that a fraction of $\mathrm{CH}_{3} \mathrm{OH}$ molecules is actually segregating into its crystalline form.

It has been pointed out previously that segregation takes place if diffusion of molecules in the ice is possible and it is energetically favorable for molecules of the same species to group together, being ice diffusion barriers proportional to their volatility (Öberg et al. 2009). Therefore, the most volatile ice species, like CO, can diffuse easily within the water ice structure and, eventually, meet other CO molecules. However, since 


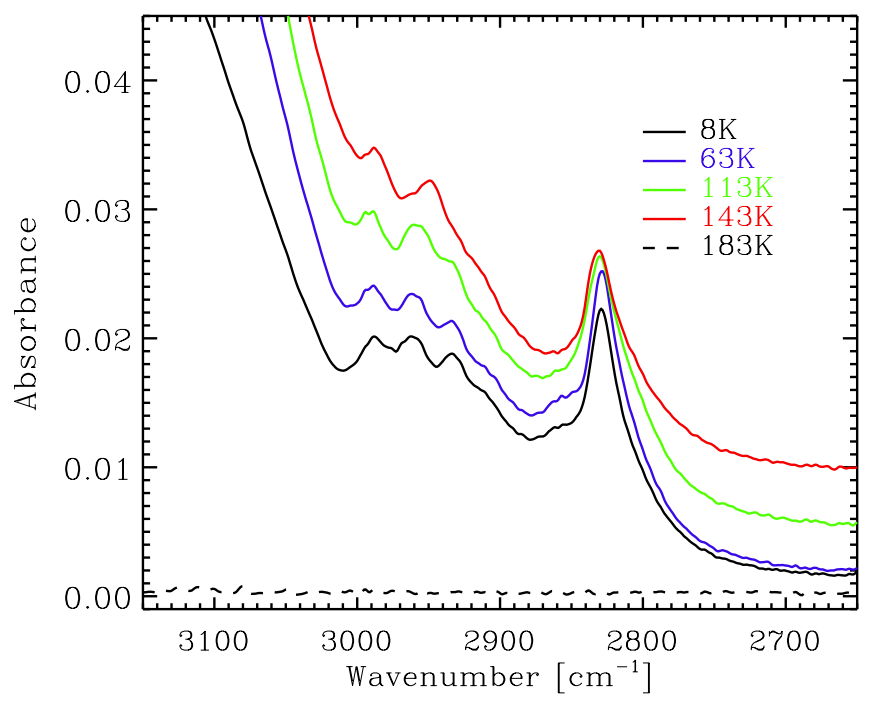

Fig. 10. Evolution of the $\mathrm{C}-\mathrm{H}$ stretching modes of $\mathrm{CH}_{3} \mathrm{OH}$ during the warm-up of a binary mixture (experiment E9). Spectra are offset for clarity.

the $\mathrm{CO}-\mathrm{CO}$ interaction is the weakest among the species studied here, only small groups of segregated $\mathrm{CO}$ molecules can be formed before they reach the ice surface and desorb or, alternatively, the segregated-CO seed is torn apart by stronger interactions with other species (mainly $\mathrm{H}_{2} \mathrm{O}$, since it is the major constituent in our pre-cometary ice analogs). On the other hand, less volatile species like $\mathrm{CH}_{3} \mathrm{OH}$ can form larger groups of segregated molecules when the ice temperature is sufficiently high, despite the fact that they do not diffuse so easily through the ice structure. Once methanol molecules meet, the $\mathrm{CH}_{3} \mathrm{OH}-\mathrm{CH}_{3} \mathrm{OH}$ interactions are stronger, since these molecules form hydrogen bonds with a similar strength to those formed by $\mathrm{H}_{2} \mathrm{O}$ molecules (Bakkas et al. 1993; note that, unlike $\mathrm{CO}, \mathrm{CO}_{2}$, and $\mathrm{NH}_{3}$, water and methanol are both liquids at standard conditions) In addition, methanol desorbs at higher temperatures compared to $\mathrm{CO}, \mathrm{CO}_{2}$, and $\mathrm{NH}_{3}$. Therefore, segregated- $\mathrm{CH}_{3} \mathrm{OH}$ seeds can grow without desorbing or being disrupted, forming larger regions of segregated $\mathrm{CH}_{3} \mathrm{OH}$ which can be detected by the FTIR spectrometer.

\section{Astrophysical implications}

The heating rate used in our experiments is fast compared to most astrophysical scenarios, as it is generally the case in experimental simulations of astrophysical processes. The desorption behavior of an ice mixture does not strongly depend on the heating rate (Sect. 3.1.1), but differences in the desorption temperatures are usually found (e.g., Collings et al. 2004).

Using the kinetic parameters for pure $\mathrm{H}_{2} \mathrm{O}$ ice desorption, Collings et al. (2004) performed a series of theoretical simulations of water ice desorption with different heating rates. The desorption peak reaches its maximum at $T \sim 160 \mathrm{~K}$ for a heating rate of $4.8 \mathrm{~K} / \mathrm{min}$, similar to the value used in our experiments and at $T \sim 105 \mathrm{~K}$ for a heating rate of $1 \mathrm{~K} /$ century, close to the heating rate in hot cores.

Thermal annealing of interstellar, circumstellar, and cometary ices takes place in three different stages of stellar evolution (see Sect. 1):

- Interstellar ice mantles covering dust grains are formed in dense molecular clouds $(T \sim 10 \mathrm{~K})$. These dust grains cycle between the dense and the diffuse interstellar medium. Ice mantles do not survive the harsh radiation conditions of the diffuse medium. These cycles proceed until dust grains are incorporated in star-forming regions.

- Circumstellar ices are heated during the star formation, as the temperature of the central object increases. Some grains undergo transient episodes of "flash-heating" lasting up to hundreds of hours according to the fluctuating X-wind model for CAIs and chondrules formation (Shu et al. 1996, 1997, 2001).

- Cometary ices are heated during late thermal evolution of comets around already formed stars, as the comet comes closer to the star during its orbit.

As it was mentioned in Sect. 2, thermal annealing of thick ice mantles, which are formed in outer regions of disks around high and low-mass protostars by grain agglomeration, is mimicked more faithfully in the TPD experiments presented in this work, due to the thickness of our ice analogs. We explain in Sect. 4.2 that our results can be also applied with some limitations to cometary ices.

\subsection{Circumstellar ices}

Chemical models of hot cores use the experimental data on TPD of ice analogs in the study of their astrochemical network (e.g., Viti et al. 2004; Wakelam et al. 2004). It was suggested that binary mixtures can be used as templates for more complex ice mixtures to model the desorption of interstellar and circumstellar ices (Fayolle et al. 2011). In our experiments, we have observed co-desorption of $\mathrm{CO}, \mathrm{CO}_{2}$, and $\mathrm{NH}_{3}$ with $\mathrm{CH}_{3} \mathrm{OH}$ in all the studied mixtures with an abundance of these species relative to water above $\sim 3 \%$ (which falls in the range of typical abundances found in most astrophysical environments; see Mumma \& Charnley 2011, and references therein). Appropiate TPD experiments with complex (at least tertiary) mixtures are thus needed to incorporate this co-desorption into the models.

The TPD curves and IR spectral evolution of our pre-cometary ice analogs allowed us to build a schematic representation of the thermal annealing process of ice mantles in circumstellar regions (Fig. 11). The heating rate applied to the precometary ice mixtures in experiments E10 and E11 is of the same order of magnitude than that applied to ice mantles during the "flash-heating", as explained by the fluctuating X-wind model for CAIs and chondrules formation. Therefore, similar desorption temperatures are expected in this case. We have scaled laboratory temperatures to the scenario in which grains at a certain distance are heated as the temperature of the central object increases by multiplying them by a factor of 0.62 . This factor corresponds to the relation between the desorption peak temperature of water ice in our experiments $(\sim 170 \mathrm{~K})$ and the one expected for a slower heating rate ( $1 \mathrm{~K} /$ century; Collings et al. 2004).

The temperature gradient generated in the circumstellar envelope by the central protostar leads to chemical segregation in the gas phase. High-angular resolution observations of protostellar envelopes represent the best chance to observe this phenomenon in space.

\subsection{Cometary ices}

The large size of a comet nucleus (up to $40 \mathrm{~km}$ in diameter) establishes several important differences between the simulations we have performed and the real processes that take place during the thermal annealing of a comet:

- Comet nuclei have a temperature gradient in their interiors and the temperature profile is not symmetric 
A\&A 564, A8 (2014)

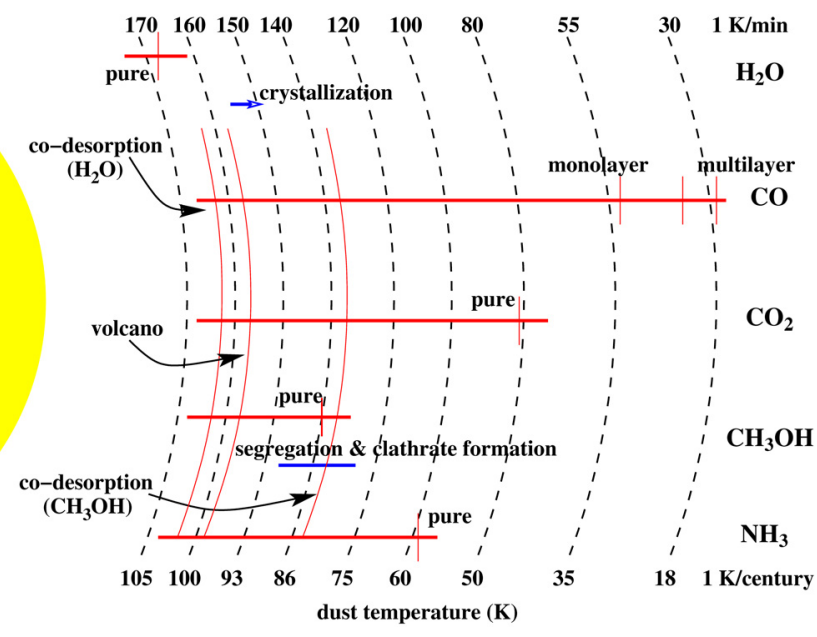

Fig. 11. Schematic representation of thermal annealing process of ice mantles in circumstellar regions based on our experimental results for the warm-up of a pre-cometary ice mixture $\left(\mathrm{H}_{2} \mathrm{O}: \mathrm{CO}: \mathrm{CO}_{2}: \mathrm{CH}_{3} \mathrm{OH}: \mathrm{NH}_{3}=100: 10-20: 10-20: 5: 5\right)$. Red lines represent the temperature ranges at which the species used in our precometary ice analogs desorb. Approximate positions of desorption peaks are indicated with vertical red lines. Desorption of $\mathrm{NH}_{3}$ and $\mathrm{CH}_{3} \mathrm{OH}$ prior to volcano desorption takes place when their abundance relative to water is above $\sim 3 \%$ in the ice. Blue lines represent the temperature ranges at which structural changes in the ice occur. The top temperature scale corresponds to a heating rate of $1-2 \mathrm{~K} / \mathrm{min}$ (used in experiments E10 and E11 with our pre-cometary ice mixtures and also suited to the "flash-heating" of dust grains during the periodic radial excursions considered in the fluctuating X-wind model for CAIs and chondrules formation). The bottom temperature scale corresponds to a first approximation to grains at a certain distance heated with a heating rate of $1 \mathrm{~K} /$ century, see text for details.

(Prialnik et al. 2008). The whole ice sample in TPD experiments is kept at the same temperature.

- The Deep Impact mission revealed that comet 9P/Temple 1 has an inhomogeneous composition at least at the surface layers (Thomas et al. 2007), while our ice analogs are expected to be homogeneously mixed.

- Molecules released from the ice can flow both outward (toward the cometary surface and the coma), or inward, due to the dynamic percolation through the comet nucleus (Notesco \& Bar-Nun 2000).

- The energy balance in a comet, dominated by the solar energy during the late thermal evolution of these objects, could be more complex than that of our experimental set-up with a small substrate and a controlled warm-up process.

In any case, this work should contribute to a better understanding of the late thermal cometary evolution, particularly, of the formation of the coma, as volatiles begin to sublime when the comet approaches the Sun during its orbit. Assuming a symmetrical temperature profile and a homogeneous ice composition in the nucleus, our simulations would correspond to the warm-up process suffered by a finite cometary slice, which is not at the same temperature than the rest of the comet nucleus during the heating process. The thermal annealing of a comet could thus be seen in a first approximation, as the sum of the warm-up of every cometary slice with a missmatch between the temperature of each slice in a certain moment. Molecules flowing inward instead of outward could alter the composition of the coma compared to that of the nucleus.

From our results, we can infer that when a comet is at a distance to the Sun close enough to heat the cometary surface to a temperature of around $20 \mathrm{~K}$, CO ice starts to desorb along with other volatile species that possess a similar desorption temperature, such as $\mathrm{N}_{2}$ and $\mathrm{O}_{2}$ (Collings et al. 2004), and the coma begins to form. As we have observed in our experiments, once the desorption of $\mathrm{CO}$ has begun, it desorbs continuously during the warm-up.

As the comet gets closer to the Sun, less volatile species like $\mathrm{CO}_{2}$ desorb successively, although in small amounts. Desorption of $\mathrm{NH}_{3}$ and $\mathrm{CH}_{3} \mathrm{OH}$ will take place if their abundance relative to water is above $\sim 3 \%$ in the cometary ice. When the temperature in the most external layer is high enough to allow desorption of, for instance, $\mathrm{CO}_{2}$, desorption of more volatile species like CO should be taking place in inner layers due to the temperature gradient of the comet nucleus. Similarly, if desorption of $\mathrm{NH}_{3}$ begins in the most external ice layer, $\mathrm{CO}_{2}$ and $\mathrm{CO}$ should be desorbing from inner layers. Therefore, the possible co-desorption of several species with $\mathrm{CH}_{3} \mathrm{OH}$ should have no strong consequences on the composition of the coma because the same species that would co-desorb with $\mathrm{CH}_{3} \mathrm{OH}$ should be also desorbing from inner layers.

The initially (small) CO-rich coma thus becomes more chemically complex as it is enriched with other less volatile molecules during the orbit of the comet. The contribution of the inner ice layers makes it difficult to predict the proportion in which volatiles are present in the coma at any time and also how this proportion varies as volatiles desorb from deeper inner layers simultaneously to the desorption of less volatile species from the outer layers. In any case, $\mathrm{CO}$ is expected to be the main component in the early stages of activity.

Once the temperature has reached the value at which phase transition between amorphous and crystalline water ice takes place, volcano desorption of all volatiles would increase the activity of the comet. After this event, desorption of water begins to be quite important, becoming the main component in the coma, as it is observed in IR and radio spectroscopic surveys (Mumma \& Charnley 2011, and references therein). We note that the detection of species in the coma also includes the "daughters" of parent ice molecules in the nucleus, making the interpretation of cometary data even more complex than outlined above.

\subsection{The ESA-Rosetta cometary mission}

Rosetta is scheduled to arrive at comet 67P/ChuryumovGerasimenko in July 2014. After that, the Rosetta orbiter will remain in close proximity to the comet nucleus and a small lander will be released onto its surface. The Rosetta orbiter includes eleven scientific instruments. The instrument ROSINA (Rosetta Orbiter Spectrometer for Ion and Neutral Analysis) will determine the composition of the comet atmosphere, playing a similar role to the QMS in our experiments. The Rosetta lander is known as Philae. There are two evolved gas analyzers (EGAs) among the ten instruments on board Philae: Ptolomey and COSAC (COmetary SAmpling and Composition experiment). The instrument COSAC is a combined gas chromatograph - mass spectrometer that will analyze the volatile fraction of surface and subsurface samples with special attention to the detection and identification of complex organic molecules. Alternatively, it can be operated in sniffing mode where only the mass spectrometer is used, analyzing the gas present in the ionization chamber (This gas is mainly composed of external gas in the case of high pressures; otherwise internal contamination dominates. Goesmann et al. 2012). While the Rosetta orbiter will probably survive perihelion passage, Philae may suffer overheating and cease functioning once the comet approaches its closest distance to the Sun. 
Table 5. Estimation of heliocentric distance, temperature, and heating rate of comet $67 \mathrm{P} /$ Churyumov-Gerasimenko during its orbit, as calculated using orbital parameters provided online by the Jet Propulsion Laboratory (NASA) and Eq. (5).

\begin{tabular}{cccc}
\hline \hline Date & $r_{\mathrm{h}}(\mathrm{UA})$ & $T(\mathrm{~K})$ & Heating rate $(\mathrm{K} / \mathrm{min})$ \\
\hline $01-07-2014$ & 3.80 & 141.0 & $6.7 \times 10^{-5}$ \\
$01-08-2014$ & 3.63 & 144.0 & $(\sim 3.0 \mathrm{~K} / \mathrm{month})$ \\
\hline $01-07-2015$ & 1.35 & 236.7 & $2.0 \times 10^{-4}$ \\
$01-08-2015$ & 1.25 & 245.7 & $(\sim 0.3 \mathrm{~K} /$ day $)$ \\
\hline
\end{tabular}

Using a simple planetary energy balance equation, it is possible to estimate a mean temperature of the comet on its surface by knowing its heliocentric distance by the time Rosetta starts the measurements and to make bold predictions of what the mass spectrometers will detect based on our laboratory results. Assuming that the comet is in radiative equilibrium, meaning that the incoming radiative energy emitted by the Sun and absorbed by the comet is equal to the radiation emitted by the comet, the temperature of its surface can be calculated as

$T=\left(\frac{L_{\mathrm{sun}}(1-a)}{16 \pi \sigma r^{2}}\right)^{1 / 4}$,

where $L_{\mathrm{sun}}$ is the Sun luminosity, $a$ the albedo of the comet, $\sigma$ the Boltzmann constant, and $r$ the heliocentric distance of the comet.

On July 1, 2014, the distance from the comet to the Sun will be at about 3.80 AU. Assuming a cometary albedo of $a=0.054$ (Kelley et al. 2009), the mean temperature at the surface of the comet will be $T \sim 141.0 \mathrm{~K}$. In our experiments with a heating rate of $1 \mathrm{~K} / \mathrm{min}$, water does not desorb substantially at this temperature. The coma would thus be composed of small amounts of more volatile species (mostly $\mathrm{CO}$, with the presence of less volatile species like $\mathrm{CO}_{2}$ and, maybe, $\mathrm{NH}_{3}$ and $\mathrm{CH}_{3} \mathrm{OH}$ ). However, the heating rate applied to the comet during its orbit around the Sun is much slower than the one applied to the ice analogs in our simulations. Data from Table 5 allows us to make a rough estimate of the heating rate during the Rosetta approach and near the perihelion after a year. As the comet gets closer to the perihelion of its orbit, its velocity, and therefore, also the heating rate, increase. Combining the theoretical simulations performed by Collings et al. (2004) of water ice desorption with different heating rates and the cometary heating rate estimated in Table 5 by the time of arrival scheduled for Rosetta, we find that the desorption peak temperature for water ice in comet 67P/Churyumov-Gerasimenko should fall between $115 \mathrm{~K}$ and $130 \mathrm{~K}$, approximately, This temperatures correspond to a heliocentric distance between 5.75 AU and 4.50 AU, according to Eq. (5). Therefore, superficial water ice should be detected already during the first measurements with an increasing activity in the next months. It is difficult, though, to predict how the composition of the coma will be affected by desorption from different layers as the comet is heated by the Sun.

Observations carried out with the Spitzer Space Telescope during the last passage of comet 67P/Churyumov-Gerasimenko did not detect signs of activity before $r_{\mathrm{h}}=4.30$ AU (Kelley et al. 2009). The authors thus expected to find an inactive or weakly active nucleus at the arrival of Rosetta. However, groundbased observations carried out at the VLT have recently revealed that the comet presented detectable activity at a heliocentric distance of $r_{\mathrm{h}}=4.30 \mathrm{AU}$, which is based on the excess flux in the $R$-band assigned to dust brightness. Therefore, the comet is expected to be active again in March 2014 before the arrival of Rosetta (Snodgrass et al. 2013), which is in line with our predictions presented above. Analysis of previous observations also indicates that the peak of activity takes place about two months after perihelion (de Almeida et al. 2009).

In addition, we expect to use the experimental data reported here as a benchmark for the analysis of the data collected by the mass spectrometers on board Rosetta as comet $67 \mathrm{P} /$ ChuryumovGerasimenko is heated during its approach to the Sun. This will shed light on the relation among the nucleus and the coma compositions, and the ice conditions. Despite the differences between these simulations and the real scenario (see Sect. 4.2), our pre-cometary ice analogs with up to five components and astrophysically relevant compositions represent the most realistic approach to the thermal annealing of a cometary ice reported to date.

\section{Conclusions}

We have studied the temperature programmed desorption (TPD) of pre-cometary ice analogs with up to five components $\left(\mathrm{H}_{2} \mathrm{O}\right.$, $\mathrm{CO}, \mathrm{CO}_{2}, \mathrm{CH}_{3} \mathrm{OH}$, and $\mathrm{NH}_{3}$ ) using mass spectrometry to detect molecules desorbing to the gas phase and complementary IR spectroscopy to study the composition, structure, and evolution of the ice during warm-up. Comparison with pure ice experiments, which are made of a single molecular component, has revealed several effects on both the TPD curves and the IR spectra arising mainly from the interaction between $\mathrm{H}_{2} \mathrm{O}$, the major constituent of our pre-cometary ice analogs, and the rest of the species, and also between $\mathrm{CH}_{3} \mathrm{OH}$ and the most volatile components.

All species present desorption peaks at temperatures near the ones corresponding to pure ices. For $\mathrm{NH}_{3}$ and $\mathrm{CH}_{3} \mathrm{OH}$, these desorption peaks are observed for the first time in water-rich ice analogs at $T \sim 97 \mathrm{~K}$ and $T$ between 140-147 K, respectively. Both species were previously classified as "water-like", since only peaks at temperatures close to water desorption were reported for co-deposited binary mixtures. Thermal desorption of ice mixture analogs with different abundances of $\mathrm{NH}_{3}$ and $\mathrm{CH}_{3} \mathrm{OH}$ have revealed that desorption in our experiments as in the pure ices is detected for ices with an abundance above $~ 3 \%$ relative to water.

Desorption of molecules from a pure ice environment suggest segregation of the species in the ice mixture to some extent. Only segregation of $\mathrm{CH}_{3} \mathrm{OH}$ molecules was confirmed by means of IR spectroscopy. The $\mathrm{C}-\mathrm{O}$ stretching band of $\mathrm{CH}_{3} \mathrm{OH}$ at $\sim 1025 \mathrm{~cm}^{-1}$ is redshifted in the pre-cometary ice mixture at $8 \mathrm{~K}$, but a blueshift is observed at temperatures above $\sim 110 \mathrm{~K}$, indicating segregation of this species and formation of a type II clathrate hydrate. Segregation of $\mathrm{CO}, \mathrm{CO}_{2}$, and $\mathrm{NH}_{3}$ molecules may occur only to a small extent due to their weaker interactions.

Desorption peaks at temperatures higher than the corresponding temperature for pure ices are also observed for all the species, except for $\mathrm{H}_{2} \mathrm{O}$, which indicates entrapment of molecules within the water ice structure. The desorption peaks at $T \sim 159 \mathrm{~K}$ correspond to volcano desorption after crystallization of the water ice structure, while the peaks at $T \sim 165 \mathrm{~K} \mathrm{re}-$ sult from co-desorption with water. The shift between the water desorption peak at $T \sim 170 \mathrm{~K}$ and the co-desorption peaks temperature can be explained by the contribution of a second volcano desorption peak in the $160-170 \mathrm{~K}$ temperature range due to the phase change from cubic to hexagonal water ice. This phase change is eased by the presence of large quantities of trapped molecules (Notesco \& Bar-Nun 2000). 
Prior to the volcano desorption, co-desorption of the more volatile species $\left(\mathrm{CO}, \mathrm{CO}_{2}\right.$, and $\left.\mathrm{NH}_{3}\right)$ with $\mathrm{CH}_{3} \mathrm{OH}$ has been detected at $T$ between $140-147 \mathrm{~K}$.

During thermal annealing of the ice analogs, $\mathrm{CO}, \mathrm{CO}_{2}$, and $\mathrm{NH}_{3}$ molecules can find themselves in ice regions where segregated methanol is the dominant component due to the further segregation of $\mathrm{CH}_{3} \mathrm{OH}$. Methanol behaves similarly to water, forming hydrogen bonds with a similar strength. Therefore, the three most volatile species co-desorb with methanol and later with water. Peaks corresponding to co-desorption of $\mathrm{CO}, \mathrm{CO}_{2}$, and $\mathrm{NH}_{3}$ with methanol cannot be reproduced in experiments with binary mixtures, which are extensively used in the study of thermal desorption of interstellar ices. In addition, IR spectra confirmed that $\mathrm{CO}$ is continuously desorbing between the desorption peaks corresponding to monolayer desorption and codesorption with methanol.

The experiments reported here help to understand the thermal annealing process of circumstellar ices inside hot cores. The heating rate applied to our ice analogs is on the same order of magnitude than the one dust grains experience during the periodic radial excursions considered in the fluctuating X-wind model for CAIs and chondrules formation (Shu et al. 1996, 1997, 2001). A similar behavior is expected for ice mantles at a given distance from the central object, as they are heated with an approximate heating rate of $1 \mathrm{~K} /$ century. However, differences in desorption peak temperatures are expected. Observations with sufficient spatial resolution will serve to test the model sketched in Fig. 4.2.

With some limitations, these results can be also applied to cometary ices. A possible scenario of ice evaporation during comet approach to the Sun is provided in Sect. 4.2. The TPD curves of a pre-cometary ice analog indicate that water could be already detected in the coma of comet 67P/ChuryumovGerasimenko during the first approach of the ESA-Rosetta cometary mission.

Acknowledgements. We are grateful to Gustavo A. Cruz Díaz, Barbara Michela Giuliano, and Antonio Jiménez Escobar for their support on the experiments. This research was financed by the Spanish MINECO under projects AYA201129375 and CONSOLIDER grant CSD2009-00038. R.M.D. benefited from a FPI grant from Spanish MINECO.

\section{References}

Attard, G., \& Barnes, C. 1998, in Surfaces (Oxford Science Publications), 72 Bar-Nun, A., Herman, G., \& Laufer, D. 1985, Icarus, 63, 317

Bakkas, N., Bouteiller, Y., Louteiller, A., Perchard, J. P., \& Racine, S. 1993, J. Chem. Phys. 99, 3335

Bisschop, S. E., Fraser, H. J., Öberg, K. I., van Dishoeck, E. F., \& Schlemmer, S. 2006, A\&A, 449, 1297

Blake, D., Allamandola, L., Sandford, S., Hudgins, D., \& Freund, F. 1991, Science, 254, 548

Bolina, A. S., Wolf, A. J., \& Brown, W. A. 2005a, J. Chem. Phys., 122, 4713
Bolina, A. S., Wolf, A. J., \& Brown, W. A. 2005b, Surface Sci., 598, 45 Brown, W. A., Viti., S., Wolff, A. J., \& Bolina, A. S. 2006, Faraday Discuss., 133,113

Collings, M. P., Dever, J. W., Fraser, H. J., \& McCoustra, M. R. 2003a, ApJ, 583, 1058

Collings, M. P., Dever, J. W., Fraser, H. J., \& McCoustra, M. R. 2003b, Ap\&SS, 385,633

Collings, M. P., Anderson, M. A., Chen, R., et al. 2004, MNRAS, 354, 1133

Cuppen, H. M., Penteado, E. M., Isokoski, K., van der Marel, N., \& Linnartz, H. 2011, MNRAS, 417, 2809

de Almeida, A. A., Sanzovo, D. T., Sanzovo, G. C., Boczko, R., \& Torres, R. M. 2009, Adv. Space Res. 43, 1993

d'Hendecourt, L. B., \& Allamandola, L. J. 1986, A\&AS, 64, 453

Dowell, L. G., \& Rinfert, A. P. 1960, Nature, 188, 1144

Eberhardt, P. 1999, Space Sci. Rev., 90, 45

Ehrenfreund, P., Kerkhof, O., Schutte, W. A., et al. 1999, A\&A, 350, 240

Falk, M., \& Whalley, E. 1961, J. Chem. Phys. 34, 1554

Fayolle, E. C., Öberg, K. I., Cuppen, H. M., Visser, R., \& Linnartz, H. 2011, ApJ, 529, A74

Fraser, H. J., Collings, M. P., McCoustra, M. R. S., \& Williams, D. A. 2001, MNRAS, 327, 1165

Goesmann, F., McKenna-Lawlor, S., Roll, R., et al. 2012, Planet. Space Sci., 66, 187

Hagen, W. 1981, Chem. Phys., 56, 367

Hagen, W., Tielens, A. G. G. M., \& Greenberg, J. M. 1983, A\&AS, 51, 389

Herbst, E., \& van Dischoeck, E. F. 2009, ARA\&A, 47, 427

Isokoski, K. 2013, Ph.D. Thesis

Jiang, G. J., Person, W. B., \& Brown, K. G. 1975, J. Chem. Phys., 62, 1201

Kelley, M. S., Wooden, D. H., Tubiana, C., et al. 2009, AJ, 137, 4633

Mumma, M. J., \& Charnley, S. B. 2011, ARA\&A, 49, 471

Muñoz Caro, G. M., Jiménez-Escobar, A., Martín-Gago, J. A., et al. 2010, A\&A, 522, A108

Noble, J. A., Congiu, E., Dulieu, F., \& Fraser, H. J. 2012, MNRAS, 421, 768

Notesco, G., \& Bar-Nun, A. 2000, Icarus, 148, 456

Öberg, K. I. 2009, Ph.D. Thesis, Universiteit Leiden

Öberg, K. I., Fayolle, E. C., Cuppen, H. C., van Dishoeck, E. F., \& Linnartz, H. 2009, A\&A, 505, 183

Pauls, T. A., Wilson, T. L., Bieging, J. H., \& Martin, R. N. 1983, A\&A, 124, 123

Prialnik, D., Sarid, G., Rosenberg, E. D., \& Merk, R. 2008, Space Sci. Rev., 138, 147

Sandford, S. A., \& Allamandola, L. J. 1990, ApJ, 355, 357

Sandford, S. A., \& Allamandola, L. J. 1993, ApJ, 417, 815

Sandford, S. A., Allamandola, L. J., Tielens, A. G. G. M., \& Valero, G. J. 1988, ApJ, 329, 498

Shu, F. H., Shang, H., \& Lee, T. 1996, Science, 271, 1545

Shu, F. H., Shang, H., Glassgold, A. E., \& Lee, T. 1997, Science, 277, 1475

Shu, F. H., Shang, H., Gounelle, M., Glassgold, A. E., \& Lee, T. 2001, ApJ, 548, 1029

Smith, R. S., Huang, C., Wong, E. K. L., \& Kay B. D. 1997, Phys. Rev. Lett., 79, 909

Snodgrass, C., Tubiana, C., Bramich, D. M., et al. 2013, A\&A, 557, A33

Thomas, P. C., Veverka, J., Belton, M. J. S., et al. 2007, Icarus, 187, 4

Viti, S., \& Williams, D. A. 1999, MNRAS, 310, 517

Viti, S., Collings, M. P., Dever, J. W., \& McCoustra, M. R. S. 2004, MNRAS, 354,1141

Wakelam, V., Caselli, P., Ceccarelli, C., Herbs, E., \& Castets, A. 2004, A\&A, 422, 159

Wyckof, S. 1982, in Comets, ed. L. L. Wilkening (Tucson: The University of Arizona Press), 20

Yamada, H., \& Person, W. B. 1964, J. Chem. Phys., 41, 2478

Zubko, V., Dwek, E., \& Arendt, R. G. 2004, ApJS, 152, 211 